\title{
The Growth and Saturation of Submesoscale Instabilities in the Presence of a Barotropic Jet
}

\author{
MEgAn A. STAMPER AND JOHN R. TAYLOR \\ Department of Applied Mathematics and Theoretical Physics, University of Cambridge, Cambridge, United Kingdom \\ BAYLOR FOX-KEMPER \\ Department of Earth, Environmental and Planetary Sciences, Brown University, Providence, Rhode Island
}

(Manuscript received 9 February 2018, in final form 10 September 2018)

\begin{abstract}
Motivated by recent observations of submesoscales in the Southern Ocean, we use nonlinear numerical simulations and a linear stability analysis to examine the influence of a barotropic jet on submesoscale instabilities at an isolated front. Simulations of the nonhydrostatic Boussinesq equations with a strong barotropic jet (approximately matching the observed conditions) show that submesoscale disturbances and strong vertical velocities are confined to a small region near the initial frontal location. In contrast, without a barotropic jet, submesoscale eddies propagate to the edges of the computational domain and smear the mean frontal structure. Several intermediate jet strengths are also considered. A linear stability analysis reveals that the barotropic jet has a modest influence on the growth rate of linear disturbances to the initial conditions, with at most a $\sim 20 \%$ reduction in the growth rate of the most unstable mode. On the other hand, a basic state formed by averaging the flow at the end of the simulation with a strong barotropic jet is linearly stable, suggesting that nonlinear processes modify the mean flow and stabilize the front.
\end{abstract}

\section{Introduction}

Submesoscales, that is, horizontal scales $O(0.1-10) \mathrm{km}$, vertical scales $O(100) \mathrm{m}$, and time scales $O(1)$ day, bridge the gap between the typically quasigeostrophic mesoscale and typically nonhydrostatic small scales where dynamics are not influenced by Earth's rotation. They have been shown to be associated with regions of enhanced vertical velocity, vorticity, and dissipation (Boccaletti et al. 2007; Capet et al. 2008; Lévy et al. 2012; Thomas et al. 2008) and are known to be almost ubiquitous in the world's oceans, particularly within the mixed layer at the ocean surface (McWilliams 2016). The weak vertical density gradients of the mixed layer and strong lateral gradients associated with ocean fronts together provide a background flow unstable to a number of unforced mixed layer instabilities (Haine and Marshall 1998; Haney et al. 2015) that may grow in the absence of external wind or wave forcing. These include submesoscale baroclinic instability (BCI; Fox-Kemper et al. 2008) and symmetric instability (SI; Bachman et al. 2017). BCI results in the formation of

Corresponding author: John R. Taylor, j.r.taylor@damtp.cam.ac.uk submesoscale eddies, while SI, a hybrid of gravitational and inertial instabilities, can result in isopycnally aligned (i.e., "slantwise") convection cells. As both submesoscale BCI and SI thrive in low stratification, these instabilities can both be categorized as types of mixed layer instability (MLI), though this term is sometimes applied preferentially to describe BCI.

Taylor et al. (2018) present a study of submesoscales in the Southern Ocean-a region for which comparatively little is known about submesoscales-motivated by in situ observations from the Surface Mixed Layer Evolution at Submesoscales (SMILES) project cruise. The study examined the extent to which the strong currents of the Antarctic Circumpolar Current (ACC) modify submesoscales generated through BCI. The nonlinear evolution of a cold, dense filament in the ACC was analyzed using numerical simulations of the top $200 \mathrm{~m}$ of the water column. These simulations demonstrated that a strong eastward barotropic jet (a jet that is depth-invariant over the mixed layer and associated with the ACC) significantly modifies submesoscales. Specifically, submesoscale eddies generated through BCI are transformed into submesoscale Rossby waves, 
stable modes with upstream phase propagation. Submesoscale Rossby waves are associated with enhanced vertical velocity, and they prevent the frontal structure from being entirely destroyed (as would be typical for BCI in the absence of a barotropic jet).

This previous work raises an important open question: How does the suppression of BCI and modification of submesoscale eddies depend on the strength of the barotropic jet? We will address this question using a combination of linear stability analysis and nonlinear numerical simulations, using a highly idealized setup representing an isolated mixed layer front collocated with a barotropic jet. Here we distinguish a mesoscale jet in geostrophic balance with the sea surface height gradient from any thermal wind shear within the mixed layer where submesoscales are most active; that is, the jet is effectively barotropic and taken as independent of the front over our domain of interest.

The phenomenon of barotropic control of BCI has received considerable attention in the atmospheric literature. Analytic studies by various authors (Kuo 1949; McIntyre 1970; Held and Andrews 1983) considered $\mathrm{BCI}$ in the presence of a small-amplitude barotropic jet. However, in our case it is clear that the observed jet magnitude is not small, having alongfront depthinvariant velocity significantly in excess of the baroclinic velocity in the mixed layer (about 1.2 and $0.1 \mathrm{~m} \mathrm{~s}^{-1}$, respectively).

Barotropic control of BCI was noted in numerical simulations of the atmosphere by Simmons and Hoskins (1978) and, later, by James and Gray (1986). James and Gray (1986) termed this the barotropic governor effect. A numerical study by James (1987), with constant barotropic shear added to a baroclinically unstable flow, indicated that linear growth rates of BCI could be substantially reduced by increasing barotropic shear.

Nakamura (1993a) verified these findings analytically using a two-layer quasigeostrophic model. Three piecewise constant regions of uniform potential vorticity (PV) were introduced to add a linear barotropic flow (or constant barotropic shear). A linear stability analysis demonstrated the same growth-rate reduction with increased shear as seen by James (1987). In addition, the solution contained momentum flux divergence at the boundaries between the uniform PV regions. These discontinuities acted to reinforce the initial barotropic shear, suggesting the existence of a nonlinear feedback process. These nonlinear effects were examined by Nakamura (1993b) using a quasigeostrophic model, which demonstrated significant convergent momentum fluxes and intensification of the barotropic jet. Each of these previous studies finds that a barotropic jet reduces BCI growth rates, in some cases substantially. In this paper we will show that a sufficiently strong barotropic jet can completely arrest submesoscale BCI.

The organization of the paper will be as follows. Section 2 describes the problem setup and formulation. Section 3 introduces the results of a series of numerical simulations, performed using a nonhydrostatic Boussinesq governing equation solver, Diablo. In section 4 , we analyze the linear stability of the initial conditions to the prescribed nonlinear barotropic flow. We separate the roles of two features of a barotropic jet-its associated shear and its effect on potential vorticity gradients - to quantify their individual influence on MLI. Finally, we evaluate the linear stability of a basic state composed of an alongfront average taken from the end of the numerical simulations, demonstrating that BCI has been arrested in the case with the strongest barotropic jet.

\section{Problem setup}

We define an isolated front using an initial buoyancy profile of the following form:

$$
b_{0}=\Delta b \tanh \left(\frac{y-\frac{L_{y}}{2}}{L_{f}}\right)+N^{2} z,
$$

where buoyancy is defined relative to an arbitrary constant density, $\Delta b$ is the frontal strength, $L_{y}$ the domain width, $L_{f}$ the frontal width, and $N^{2}$ a constant stratification. The front is in thermal-wind balance with downfront velocity given by

$$
u_{W}=-\frac{\Delta b}{f L_{f}} \operatorname{sech}^{2}\left(\frac{y-\frac{L_{y}}{2}}{L_{f}}\right)\left(z-\frac{L_{z}}{2}\right),
$$

where $f$ is the Coriolis parameter and $L_{z}$ the domain height. Note that in the SI literature, this velocity is called the "geostrophic" velocity. Here, as other flow components are also largely geostrophic, the term "thermal wind velocity" is preferred. This setup is represented schematically in Fig. 1. An additional barotropic (i.e., independent of $z$ ) jet of the form,

$$
u_{\mathrm{BT}}=\Delta U_{\mathrm{BT}} \cos \left(\frac{y-\frac{L_{y}}{2}}{\frac{L_{y}}{2}} \pi\right),
$$

is added to the thermal wind.

Associated with the barotropic jet and thermal wind are cross-frontal variations in shear and potential 


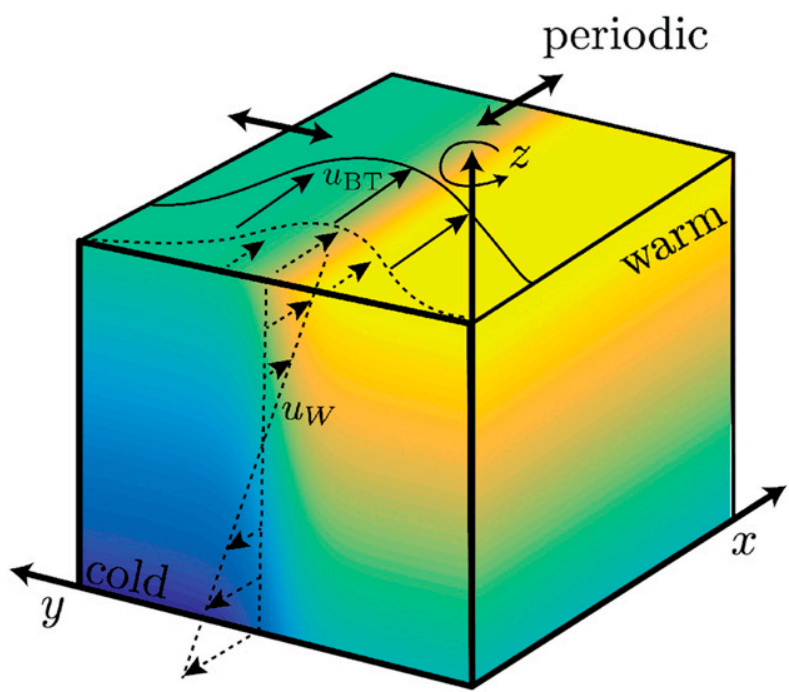

FIG. 1. A schematic representation of the problem configuration with a tanh buoyancy profile in $y$ balanced by a thermal wind $u_{W}$. Note that a vertical stratification is included in the stability analysis but not in the initial conditions of the numerical simulations.

vorticity. We denote the potential vorticity associated with the barotropic jet as

$$
\begin{aligned}
q_{B T}= & \left(f \hat{\mathbf{k}}+\nabla \times u_{\mathrm{BT}} \mathbf{i}\right) \cdot \nabla b=\left(f \hat{\mathbf{k}}+\left(2 \pi / L_{y}\right)\right. \\
& \left.\times \Delta U_{\mathrm{BT}} \sin \left\{\left[\left(y-L_{y} / 2\right) /\left(L_{y} / 2\right)\right] \pi\right\}\right) N^{2},
\end{aligned}
$$

and, for the thermal wind, $q_{W}=\left(f \hat{\mathbf{k}}+\nabla \times u_{W} \mathbf{i}\right) \cdot \nabla b$, respectively, taking care to note that $q=q_{\mathrm{BT}}+q_{W}-f N^{2} \neq$ $q_{\mathrm{BT}}+q_{W}$.

We consider an idealized representation of the ocean mixed layer with stress-free rigid lids at $z=H$, representing the ocean-atmosphere interface, and at $z=0$, representing the base of the mixed layer. The buoyancy field is decomposed according to

$$
b_{T}=b(x, y, z, t)+M^{2} y,
$$

where $b_{T}$ is the total buoyancy, and $M^{2}=\Delta b / L_{y}$. Periodic boundary conditions are applied to $\mathbf{u}$ and $b$ in both horizontal directions. The periodic boundary conditions on $b$ imply that the buoyancy change across the domain $\Delta b$ is constant in time. However, since we initialize with a localized front, this condition will not restrict the evolution of the front until buoyancy perturbations spread across the domain width.

\section{Numerical simulations}

\section{a. Setup}

We examine the influence of a barotropic jet on BCI of an isolated front by performing four three-dimensional simulations, varying the amplitude of the barotropic jet in each case such that $\Delta U_{\mathrm{BT}}=0,0.1,0.3$, and $0.6 \mathrm{~m} \mathrm{~s}^{-1}$. Parameter choices for the front are motivated by the observations made during the SMILES cruise. Specifically, we take $\Delta b=2.5 \times 10^{-4} \mathrm{~m} \mathrm{~s}^{-2}, f=-1.1875 \times 10^{-4} \mathrm{~s}^{-1}$, and $L_{f}=1500 \mathrm{~m}$. The top panel of Fig. 2 shows the crossfront buoyancy profile at the top surface, $z=120 \mathrm{~m}$. The second panel of Fig. 2 shows an example of surface $u_{W}, u_{\mathrm{BT}}$, and $u$ for a barotropic jet of strength $\Delta U_{\mathrm{BT}}=$ $0.6 \mathrm{~m} \mathrm{~s}^{-1}$. Finally, small-amplitude, random white noise perturbations of amplitude $1 \times 10^{-7} \mathrm{~m} \mathrm{~s}^{-1}$ are added to seed instability. The bottom two panels of Fig. 2 show the cross-front shear and potential vorticity gradients associated with the thermal wind (orange) and barotropic jet (blue), respectively (again, for example, with $\left.\Delta U_{\mathrm{BT}}=0.6 \mathrm{~m} \mathrm{~s}^{-1}\right)$.

Our domain height, $L_{z}=120 \mathrm{~m}$, corresponds to the observed mixed layer depth, and the domain width, $L_{x}=L_{y}=50 \mathrm{~km}$, is chosen to ensure the domain is large enough to capture several multiples of the fastestgrowing BCI mode (see section 4). Thus, the simulations allow merging and interaction of submesoscales and associated upscale energy transfer. The large horizontal extent will be particularly important in ascertaining whether a given barotropic jet strength is sufficient to explain confinement of submesoscale activity to a region close to the front. Note that while the imposed vertically invariant jet is barotropic in this setting, a low-mode mesoscale baroclinic jet with a vertical scale much deeper than the mixed layer depth would be similarly represented in the context of mixed layer submesoscales. Finally, we began each simulation with $N^{2}=0$.

Simulations are performed using Diablo, which solves the nonhydrostatic, Boussinesq governing equations (Taylor 2008). A pseudospectral method is used in both horizontal directions, and a second-order finite difference method is applied in the vertical direction. Time stepping is implemented using an implicit CrankNicolson scheme for viscous terms and an explicit lowstorage, third-order Runge-Kutta scheme for all other terms. The simulations discussed have 128 vertical grid points and 512 horizontal grid points in both $x$ and $y$ directions, implying vertical resolution of about $1 \mathrm{~m}$ and horizontal resolution of about $100 \mathrm{~m}$. As shown in Bachman and Taylor (2014), this horizontal resolution is sufficient to adequately resolve SI in a layer of this depth. The horizontal resolution being much coarser than the vertical resolution, it is necessary to define anisotropic eddy viscosities $\nu_{H}$ and $\nu_{V}$, where subscripts $H$ and $V$ denote horizontal and vertical quantities, respectively. Values of $\nu_{H}=1 \mathrm{~m}^{2} \mathrm{~s}^{-1}$ and $\nu_{V}=5 \times$ $10^{-5} \mathrm{~m}^{2} \mathrm{~s}^{-1}$ were used, ensuring that, throughout each simulation, grid spacing was less than approximately 

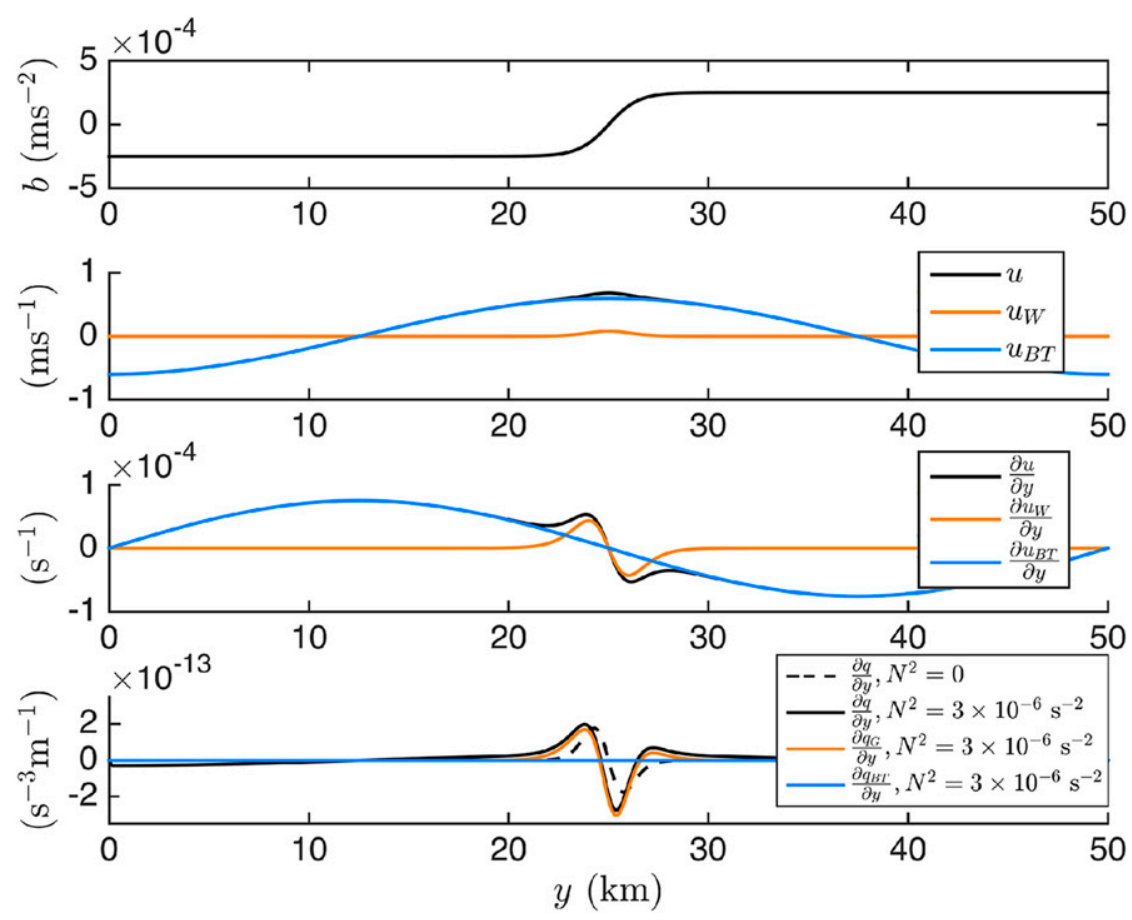

FIG. 2. Cross-front profiles of (top to bottom) buoyancy $b$, alongfront velocity $u$, velocity shear in the cross-front direction $\partial u / \partial y$, and PV gradient $\partial q / \partial y$ at the top surface $(z=0)$. The contributions from the surface thermal wind $u_{W}$, barotropic jet $u_{\mathrm{BT}}$, and total initial alongfront velocity $u$, with $\Delta U_{\mathrm{BT}}=0.6 \mathrm{~m} \mathrm{~s}^{-1}$, are also shown in the middle panels. The dashed line in the bottom panel indicates the initial PV gradient with $N^{2}=0$, as for the nonlinear simulations (note that at this stage, the only contribution to PV gradients is from the thermal wind component $u_{W}$ ), while the solid lines indicate the total, thermal wind, and barotropic components with $N^{2}=3 \times 10^{-6} \mathrm{~s}^{-2}$, as for the linear stability analysis in section 4 .

twice the Kolmogorov scale in both horizontal and vertical directions:

$$
\eta_{H, V}=\left(\frac{\nu_{H, V}^{3}}{\varepsilon}\right)^{1 / 4},
$$

where $\nu$ is the eddy viscosity and $\varepsilon$ is the viscous dissipation rate of kinetic energy calculated directly from the simulations. The diffusivity used in the buoyancy equation matches the viscosity; that is, $\kappa_{H}=\nu_{H}$ and $\kappa_{V}=\nu_{V}$. The eddy viscosity and diffusivity can be interpreted as being those associated with unresolved turbulence in the mixed layer, with the choice of Prandtl number $(\operatorname{Pr}=\nu / \kappa)$ consistent with this interpretation. Constant viscosity and diffusivity were chosen to simplify the linear stability analysis and analysis of the numerical simulations.

\section{b. General description}

Here, we begin by describing the general features of the numerical simulations. As will be shown, all simulations contain an initial period of SI that is relatively insensitive to the presence of the barotropic jet, followed by a period of $\mathrm{BCI}$ and nonlinear evolution where the barotropic jet has a much stronger influence. A detailed description of the flow during the SI and BCI phases will be given below in sections $3 \mathrm{c}$ and $3 \mathrm{~d}$, respectively.

Figure 3 a shows the evolution of the domain-averaged eddy kinetic energy, $\overline{\mathrm{EKE}}^{x y z}=1 / 2 \overline{\left(u^{\prime 2}+v^{\prime 2}+w^{\prime 2}\right)^{x y z}}$, where $\overline{(\cdot)^{x y z}}$ denotes a volume average and primes denote departures from a horizontal mean. The case with $\Delta U_{\mathrm{BT}}=0.6 \mathrm{~m} \mathrm{~s}^{-1}$ is closest to the observed barotropic jet strength (the full jet amplitude being $2 \Delta U_{\mathrm{BT}}=1.2 \mathrm{~m} \mathrm{~s}^{-1}$ ). All simulations begin with a period of $\overline{\mathrm{EKE}}^{x y z}$ growth, from 1.5 to 1.8 days, associated with SI and with very little variation between simulations with different barotropic jet strengths.

In all cases, SI is followed by inertial oscillations with a period of approximately $2 \pi /|f| \approx 14.5 \mathrm{~h}$. Inertial oscillations were also observed following SI in Taylor and Ferrari (2009), while Thomas et al. (2016) found that inertial oscillations modulate the growth rate associated with SI. Following these oscillations, each simulation experiences a second period of growth, beginning at 
(a)

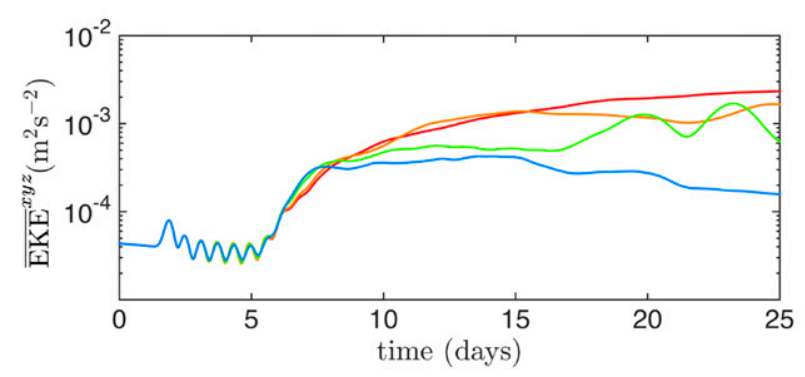

(b)

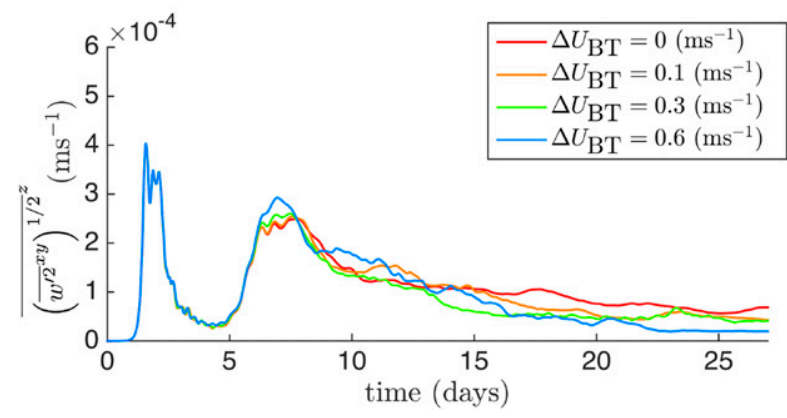

FIG. 3. (a) Domain-average EKE and (b) rms vertical velocity.

$t=5-6$ days. In the case with $\Delta U_{\mathrm{BT}}=0$ (red line), the $\overline{\mathrm{EKE}}^{x y z}$ increases until the end of the simulation, consistent with sustained conversion of potential energy into eddy kinetic energy (Fox-Kemper et al. 2008) and a frontal width (Fox-Kemper et al. 2011; Callies and Ferrari 2018a). In contrast, when $\Delta U_{\mathrm{BT}}=0.6 \mathrm{~m} \mathrm{~s}^{-1}$, $\overline{\mathrm{EKE}}^{x y z}$ saturates at about $t=7.5$ days before decaying in the late stages of the simulation.

The two phases of instability can also be distinguished through the domain-averaged root-mean-square (rms)

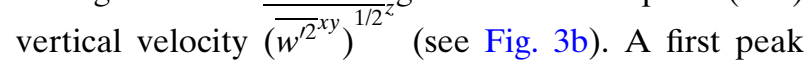
occurs in all simulations at about $t=1.5$ days, during the brief period of SI, followed by a second peak at about 7 days during a period of BCI. After the second local maximum, the rms vertical velocity decays slowly throughout the remainder of the simulations.

Horizontal slices of the vertical velocity near the lower boundary $(z=5 \mathrm{~m})$ and surface buoyancy at the top surface $(z=120 \mathrm{~m})$ are shown in Figs. 4 and 5, respectively. Changes to the buoyancy at early times are difficult to see and are excluded from Fig. 5. After 2.7 days in the simulation with $\Delta U_{\mathrm{BT}}=0$ (see Fig. $4 \mathrm{a}$ ), the vertical velocity exhibits regularly spaced bands, about $500 \mathrm{~m}$ in width, independent of the alongfront direction and characteristic of SI. By 3.8 days alongfront variations in the vertical velocity first become visible. The vertical velocity is similar in the case with $\Delta U_{\mathrm{BT}}=0.6 \mathrm{~m} \mathrm{~s}^{-1}$ during the SI phase.
At $t=7$ days, breaking baroclinic waves are visible in the vertical velocity and buoyancy fields (see Figs. 4c and 5a). Differences between the simulations with $\Delta U_{\mathrm{BT}}=0$ and $\Delta U_{\mathrm{BT}}=0.6 \mathrm{~m} \mathrm{~s}^{-1}$ are now apparent, with somewhat more regular baroclinic waves in the latter case. In both cases, narrow bands of upwelling appear on the edges of the baroclinic waves.

At later times, the simulations with $\Delta U_{\mathrm{BT}}=0$ and $\Delta U_{\mathrm{BT}}=0.6 \mathrm{~m} \mathrm{~s}^{-1}$ become drastically different. After 18 days, in the case with $\Delta U_{\mathrm{BT}}=0.6 \mathrm{~m} \mathrm{~s}^{-1}$, the front remains intact and confined to a region within about $6 \mathrm{~km}$ of the original frontal center (see Fig. 5d). The vertical velocity is similarly confined, with the largest vertical circulations near the bottom of the domain appearing on the warm side of the front at approximately $y=35 \mathrm{~km}$ (see Fig. 4f).

In contrast, when $\Delta U_{\mathrm{BT}}=0$, coherent submesoscale eddies develop and merge, with larger-scale eddies dominant in the surface buoyancy by 11 days (not shown). This results in buoyancy variations stretching much farther away from the original location of the front center, $y=25 \mathrm{~km}$. Eddy merging continues until, by 18 days, buoyancy variations have reached the boundaries of the domain, particularly on the cold side, and what remains of the original front has become very convoluted and extended in length (see Fig. 5c).

\section{c. SI}

The initial condition, with $N^{2}=0$, has regions where the potential vorticity takes the opposite sign from the Coriolis parameter (i.e., $f q<0$ ), hence meeting the criterion for SI (Hoskins 1974). The most unstable mode of inviscid SI is characterized by along-isopycnal motion in the cross-front, vertical plane (Stone 1966; Taylor and Ferrari 2009). Figure 6a shows isopycnals (dashed) and vertical velocity (color) in the simulation with $\Delta U_{\mathrm{BT}}=0.6 \mathrm{~m} \mathrm{~s}^{-1}$, consistent with mature SI circulations [cf. with Fig. 3a in Stamper and Taylor (2017) or Figs. 7 and 9 of Haney et al. (2015)].

In all simulations two distinct steps develop in the surface buoyancy that are approximately equidistant from the center of the front, each with a similar magnitude of change in buoyancy (see Fig. 6b). These steps are reminiscent of the steps that appeared in the simulations of Stamper and Taylor (2017), where they were attributed to frontogenesis induced by SI cells. For $\Delta U_{\mathrm{BT}}=0$, the main difference between the simulations of Stamper and Taylor (2017) and here is the presence of a variable lateral buoyancy gradient in the initial conditions. This constrains SI and its associated density steps to the center of the domain in $y$.

There is little variation in the growth rate of SI as $\Delta U_{\mathrm{BT}}$ is varied, evidenced by the similar eddy kinetic energy evolution for each simulation during the SI phase (see Fig. 3). However, there are small changes to the growth 
(a)

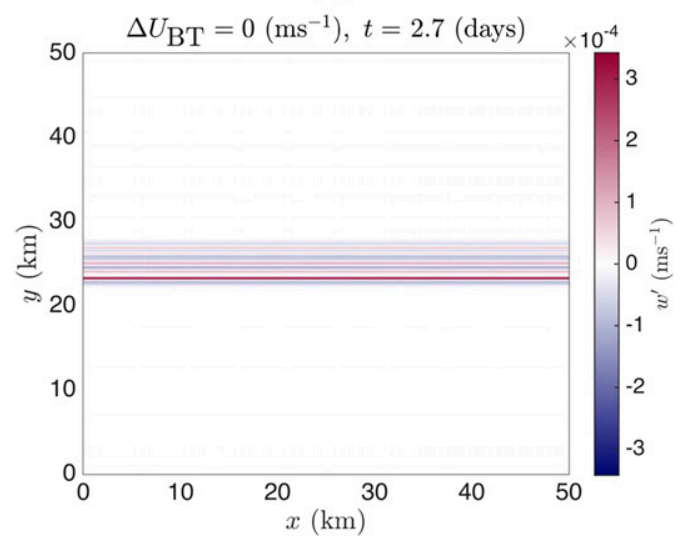

(c)

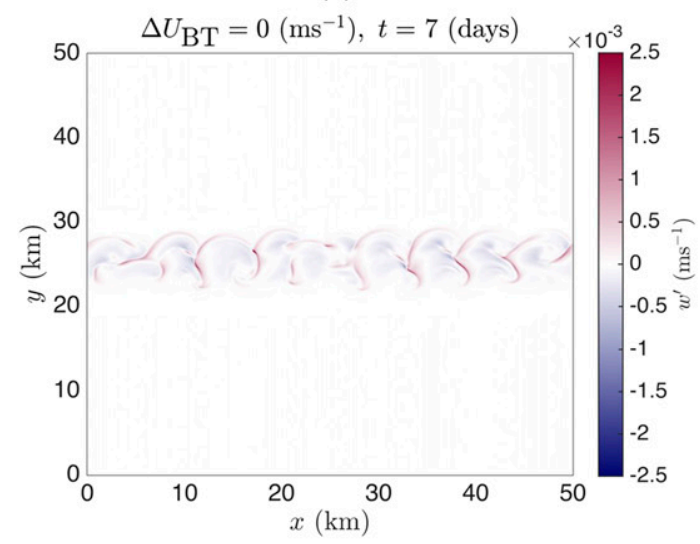

(e)

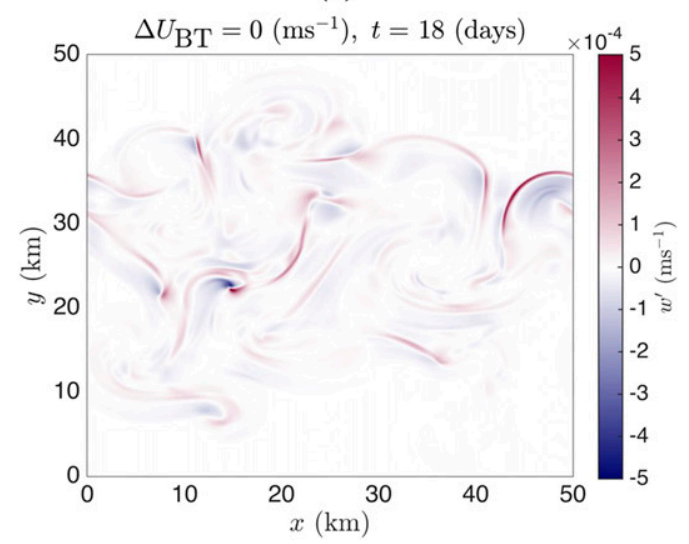

(b)

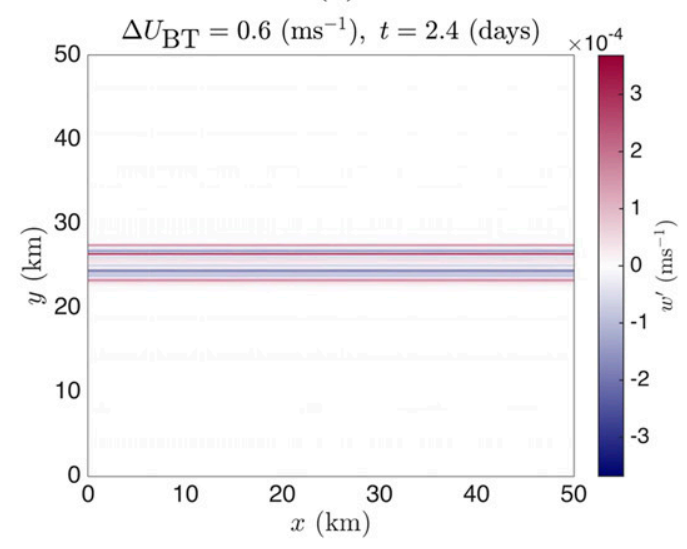

(d)

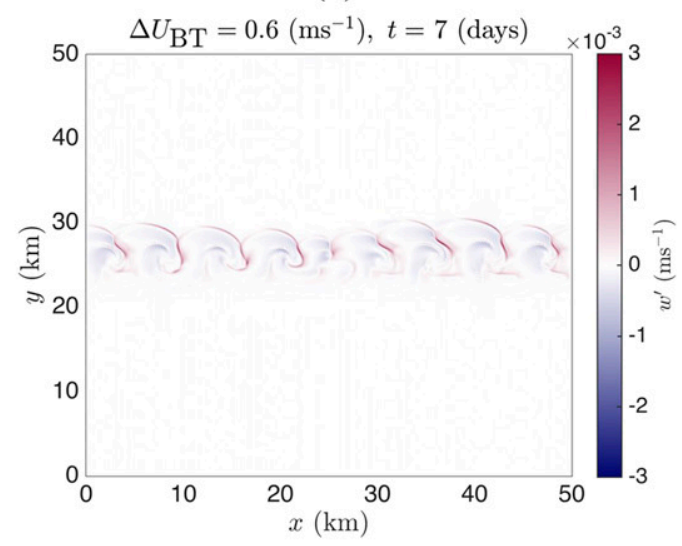

(f)

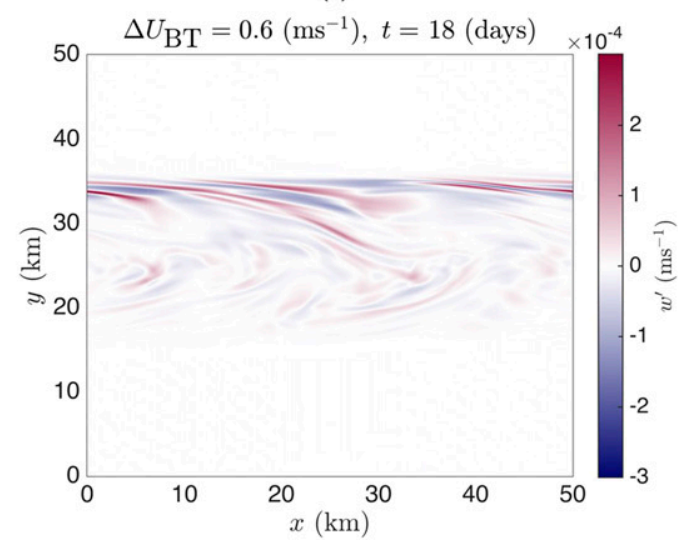

FIG. 4. Vertical velocity, $5 \mathrm{~m}$ from the bottom of the domain, at various times in the (a),(c),(e) $\Delta U_{\mathrm{BT}}=0$ and (b),(d),(f) $\Delta U_{\mathrm{BT}}=0.6 \mathrm{~m} \mathrm{~s}^{-1}$ simulations.

rate associated with SI induced by the barotropic jet. The addition of a barotropic jet creates an asymmetry in the growth of SI on the warm (anticyclonic) and cold (cyclonic) sides of the front. This can be shown by briefly revisiting the linear stability analysis of Stone (1966) and Stamper and Taylor (2017) but with the addition of a barotropic jet.
For simplicity, we will take the horizontal buoyancy gradient and the horizontal shear to be constant on the scale of the growing perturbations. Although not strictly valid here, this assumption greatly simplifies the analysis. Taking normal mode perturbations of the form 
(a)

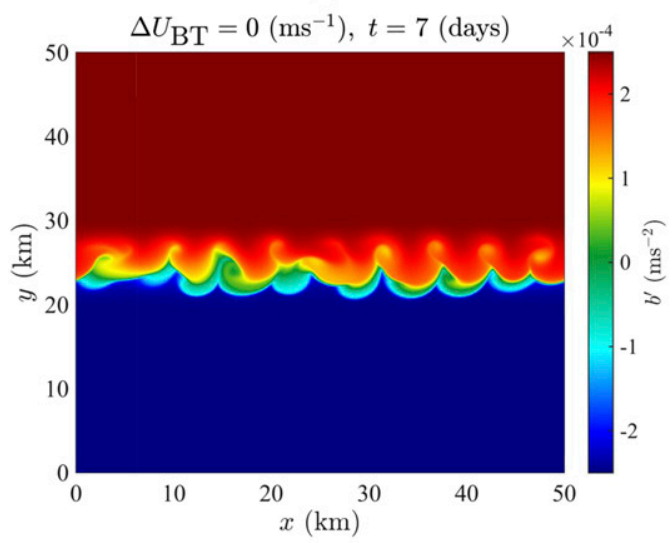

(c)

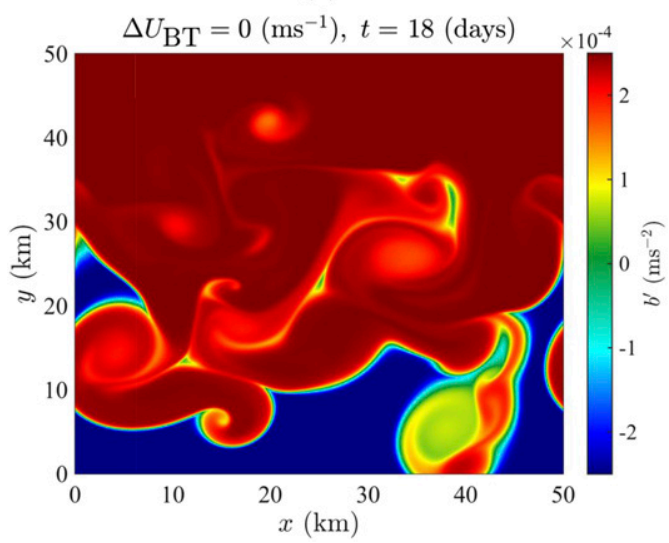

(b)

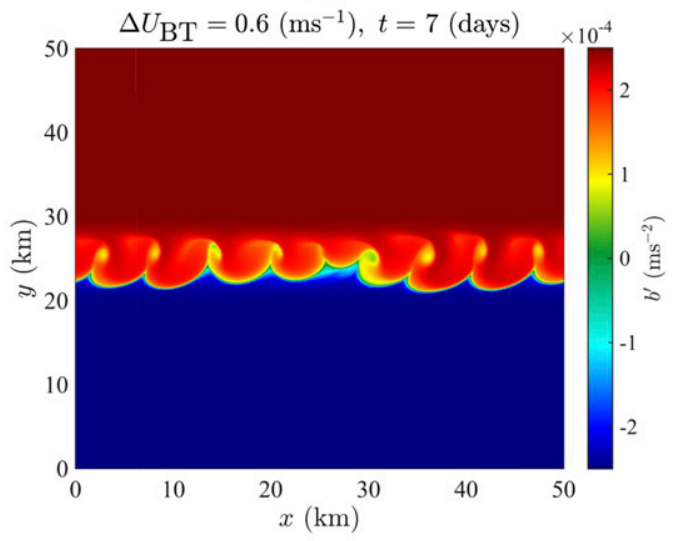

(d)

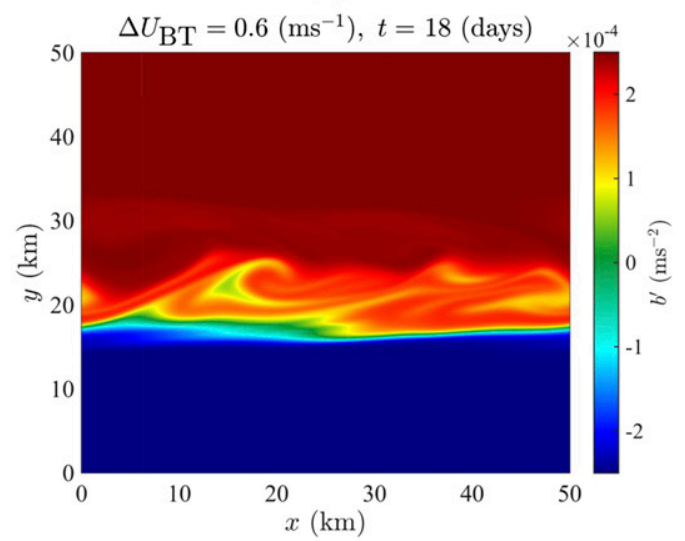

FIG. 5. Surface buoyancy at various times for (a),(c) $\Delta U_{\mathrm{BT}}=0$ and (b),(d) $\Delta U_{\mathrm{BT}}=0.6 \mathrm{~m} \mathrm{~s}^{-1}$.

$$
\left(u^{\prime}, v^{\prime}, w^{\prime}, b^{\prime}, \phi^{\prime}\right)=(\hat{u}, \hat{v}, \hat{w}, \hat{b}, \hat{\phi}) e^{i(k x+\ell y+m z)+\sigma t},
$$

linearizing, and eliminating variables algebraically from the governing equations, the growth rate for SI modes (with $k=0$ ) is

$$
\begin{aligned}
\sigma= & {\left[\frac{M^{4}}{N^{2}}-f^{2}-N^{2}\left(\frac{\ell}{m}-\frac{M^{2}}{N^{2}}\right)^{2}+f \frac{\partial u_{\mathrm{BT}}}{\partial y}\right]^{1 / 2} } \\
& +\nu\left(\ell^{2}+m^{2}\right) .
\end{aligned}
$$

This suggests that SI has larger growth rates in regions of strong anticyclonic vorticity, that is, where $\zeta_{\mathrm{BT}}=$ $\left(\nabla \times u_{\mathrm{BT}} \mathbf{i}\right) \cdot \mathbf{k}=-\partial u_{\mathrm{BT}} / \partial y>0$ in the Southern Hemisphere. Noting from Fig. 2 that the relative vorticity is anticyclonic for $y>L_{y} / 2$, we anticipate that this region will be more unstable to SI. We define the following split of the EKE between the two halves of the domain in the $y$ direction:

$$
\overline{\mathrm{EKE}}_{\mathrm{split}}^{x y z}=\overline{\operatorname{EKE}}_{y>L_{y} / 2}^{x z}-\overline{\operatorname{EKE}}_{y<L_{y} / 2}^{x z},
$$

where, for example, $\overline{\operatorname{EKE}}_{y<L_{y} / 2}^{x z}=1 / L_{x} L_{y} L_{z} \int_{0}^{L_{x}} \int_{0}^{L_{z}} \int_{0}^{L_{y} / 2}$ $\left(u^{\prime 2}+v^{\prime 2}+w^{\prime 2}\right) d y d z d x$. We anticipate that $\overline{\operatorname{EKE}}_{\text {split }}^{x y z}>0$, with more asymmetry for larger $\Delta U_{\mathrm{BT}}$. This is supported by the simulation data. Figure $6 \mathrm{c}$ shows $\overline{\mathrm{EKE}}_{\text {split }}^{x y}$ for each simulation during symmetric growth, indicating significantly higher positive values of $\overline{\mathrm{EKE}}_{\text {split }}^{x y}$ for higher values of $\Delta U_{\mathrm{BT}}$. In other words, SI is enhanced in regions of anticyclonic barotropic relative vorticity; $\zeta_{\mathrm{BT}}=$ $\left(\nabla \times u_{\mathrm{BT}} \mathbf{i}\right) \cdot \mathbf{k}=-\partial u_{\mathrm{BT}} / \partial y>0$ in the Southern Hemisphere.

\section{d. $B C I$}

The second period of $\overline{\mathrm{EKE}}^{x y z}$ growth beginning at about 5 days (see Fig. 3a) is much more strongly influenced by the barotropic jet than that during the SI phase. This second period of growth is associated with a positive volume-averaged buoyancy flux $\overline{b^{\prime} w^{\prime \prime}} x z$, indicative of BCI (Stone 1972) (Fig. 7a). The buoyancy flux is relatively unaffected by the barotropic jet until about day 6 , while after about day 8 the buoyancy flux is generally smaller in simulations with stronger barotropic jets. This implies a suppression of the extraction of 
(a)

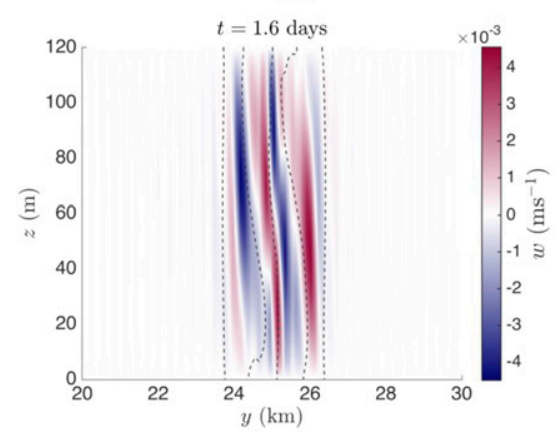

(b)

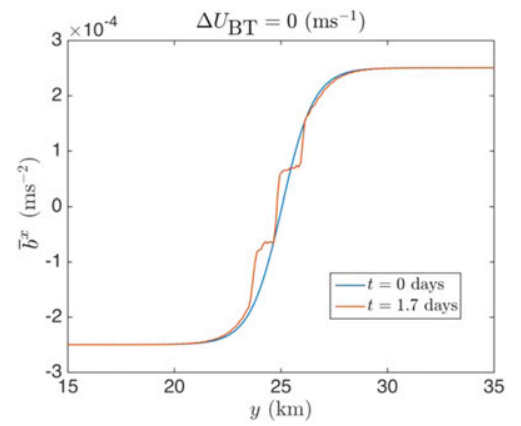

(c)

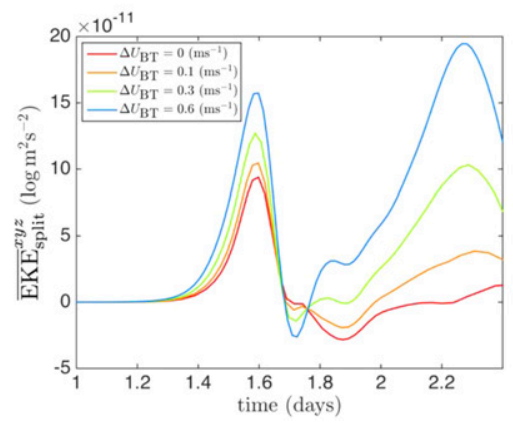

FIG. 6. (a) A cross-front section from the simulation with $\Delta U_{\mathrm{BT}}=0.6 \mathrm{~m} \mathrm{~s}^{-1}$ at $\mathrm{t}=1.6$ days, where color indicates vertical velocity $w$ and contours are isopycnals. (b) Alongfront averaged buoyancy $\bar{b}^{x}$ for the inital time (blue) and 1.7 days (red) at $z=120 \mathrm{~m}$ for $\Delta U_{\mathrm{BT}}=0 \mathrm{~m} \mathrm{~s}^{-1}$. (c) The difference between EKE for $y>L_{y} / 2$ and $y<L_{y} / 2$ [as defined in Eq. (8)] during the period of symmetric instability.

potential energy by BCI in cases with strong barotropic jets. By the end of the simulations the buoyancy flux remains elevated for the $\Delta U_{\mathrm{BT}}=0$ case, while the buoyancy flux is nearly zero for $\Delta U_{\mathrm{BT}}=0.6 \mathrm{~m} \mathrm{~s}^{-1}$.

The influence of the barotropic jet on the buoyancy flux [and hence the conversion of potential to kinetic energy, per Fox-Kemper et al. (2008)] is also reflected in the mean vertical stratification. Figure $7 b$, shows the domain-averaged vertical buoyancy gradient $\bar{N}^{x y z}=$ $\overline{\partial b / \partial z}^{x y z}$. There is a small increase in ${\overline{N^{2}}}^{x y z}$ during the growth of SI, with little variation between the simulations. In contrast, there is a second, much more significant increase in $\bar{N}^{2} x z$ associated with the onset of BCI at around 6.5 days in each case. After about 8 days the simulations with $\Delta U_{\mathrm{BT}}>0.1 \mathrm{~m} \mathrm{~s}^{-1}$ diverge significantly from the case with $\Delta U_{\mathrm{BT}}=0$. Restratification slows toward the latter stages of these simulations, with $\bar{N}^{2} x y z$ becoming steady in the $\Delta U_{\mathrm{BT}}=0.6 \mathrm{~m} \mathrm{~s}^{-1}$ case after around 15.5 days. This demonstrates that the arrest of $\mathrm{BCI}$ in this case has halted mixed layer restratification. $\mathrm{BCI}$ in the highest jet strength simulations has been unable to extract as much energy from the potential energy associated with tilted isopycnals at the front. This is also reflected in the evolution of the integrated potential energy, $E_{P}(t)=\iiint z b^{\prime} d x d y d z$ (see Fig. 7c). At 27 days, there is significantly more potential energy remaining in the system in the case with the strong barotropic jet, $\Delta U_{\mathrm{BT}}=0.6 \mathrm{~m} \mathrm{~s}^{-1}$, nearly 5 times that for the case with no barotropic jet, $\Delta U_{\mathrm{BT}}=0$.

During the late stages of $\mathrm{BCI}$ and the subsequent nonlinear evolution in the simulation with the strongest barotropic jet $\left(\Delta U=0.6 \mathrm{~m} \mathrm{~s}^{-1}\right)$, the character of the submesoscale structures is dramatically altered (cf. Figs. $5 \mathrm{c}$ and $5 \mathrm{~d}$ ). In this case, the resulting surface buoyancy profile has disturbances confined between approximately $y=35 \mathrm{~km}$ on the warm side of the front and $y=15 \mathrm{~km}$ on the cold side. At $y=15 \mathrm{~km}$, a sharp front persists in the surface buoyancy.

In simulations with large-amplitude barotropic jets, the front and submesoscale disturbances remain confined to a narrower region around the original frontal location than in the case with no barotropic jet. Figure 8 shows Hovmöller plots of buoyancy, averaged in $x$ and $z$, as a function of time and cross-front distance $y$. In the

(a)

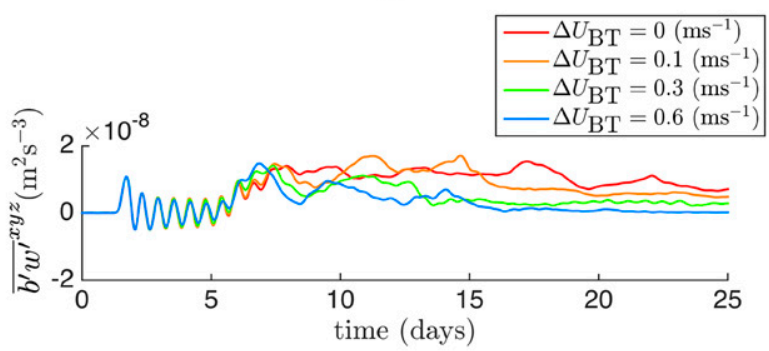

(b)

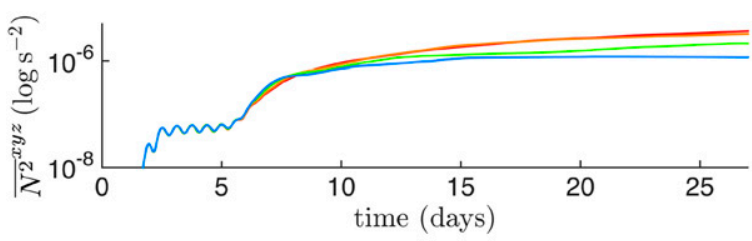

(c)

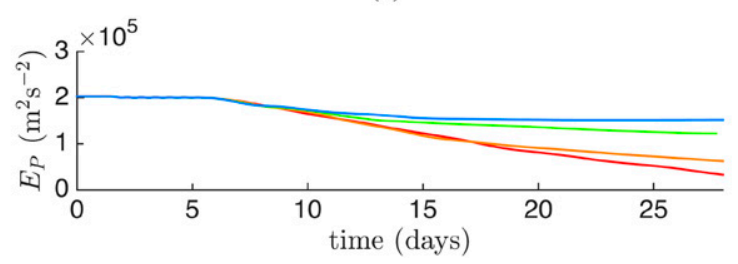

FIG. 7. Evolution of domain-averaged (a) buoyancy flux, (b) stratification, and (c) potential energy. 
(a)

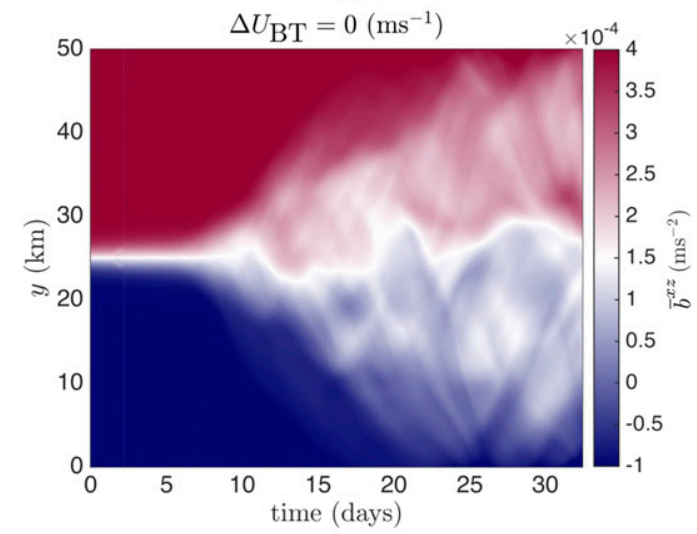

(c)

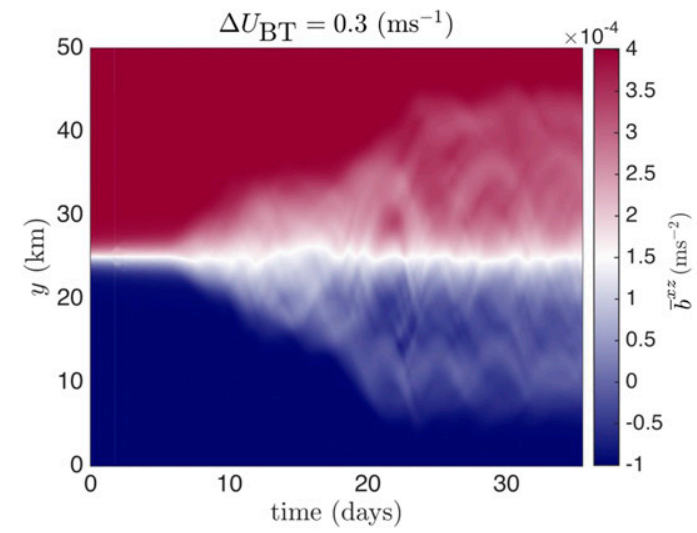

(b)

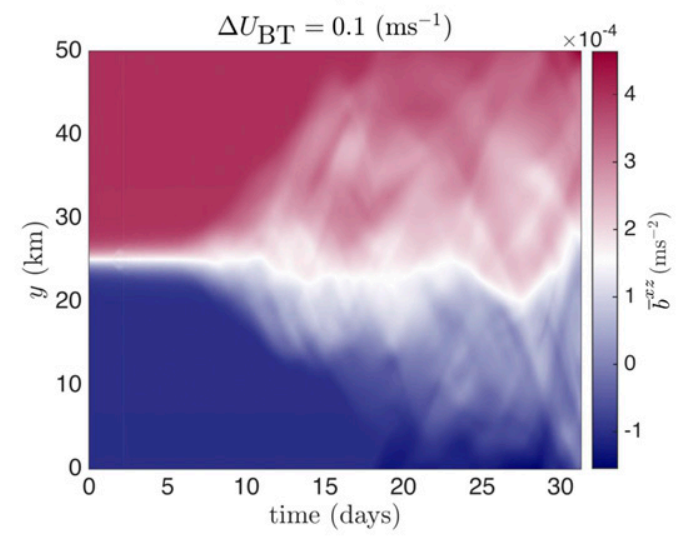

(d)

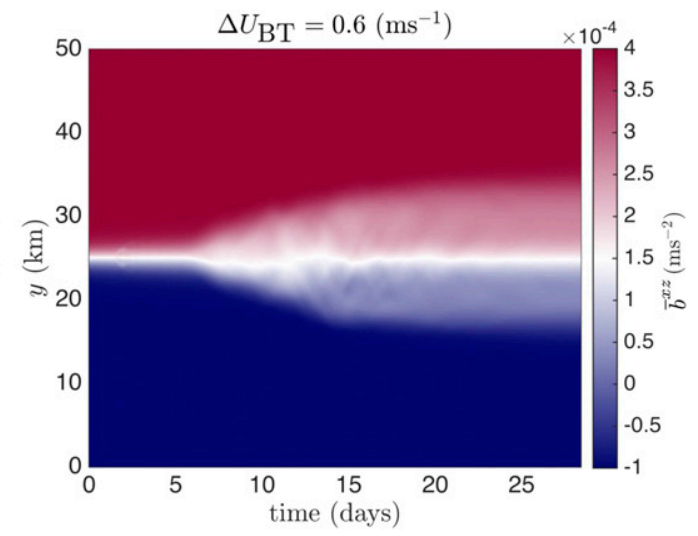

FIG. 8. Alongfront and depth averaged buoyancy $\bar{b}^{x z}$ for a variety of barotropic jet magnitudes: (a) $\Delta U_{\mathrm{BT}}=0$, (b) $\Delta U_{\mathrm{BT}}=0.1$, (c) $\Delta U_{\mathrm{BT}}=0.3$, and (d) $\Delta U_{\mathrm{BT}}=0.6 \mathrm{~m} \mathrm{~s}^{-1}$.

case without a barotropic jet, $\Delta U_{\mathrm{BT}}=0$, variations in the surface buoyancy extend across the full cross-frontal extent of the domain by about day 20 . In contrast, the surface fronts for the $\Delta U_{\mathrm{BT}}=0.3$ and $\Delta U_{\mathrm{BT}}=0.6 \mathrm{~m} \mathrm{~s}^{-1}$ cases are more confined. The $\Delta U_{\mathrm{BT}}=0.6 \mathrm{~m} \mathrm{~s}^{-1}$ simulation, in particular, appears to have reached an approximate equilibrium with little change in the frontal width from approximately day 20 onward.

Vertical circulations are similarly confined to a relatively narrow region around the front in the simulations with stronger barotropic jets. Figure 9 shows the $x$-averaged root-mean-square vertical velocity $\frac{1}{w^{12}} x^{1 / 2}$. The top two panels, at the time of the second local maxima of full domain root-mean-square vertical velocity (as can be seen in Fig. 3b), demonstrate that high vertical velocities associated with $\mathrm{BCI}$ occur near the center of the front. The values of $\overline{w^{12}} x^{1 / 2}$ at the center of the front are an order of magnitude larger than the domain-averaged root-mean-square vertical velocities $\overline{\left({\overline{w^{12}}}^{x y}\right)^{1 / 2}}$. In the $\Delta U_{\mathrm{BT}}=0.6 \mathrm{~m} \mathrm{~s}^{-1}$ case (Fig. 9b), we see that vertical velocities are already more confined in $y$ at this time compared to the $\Delta U_{\mathrm{BT}}=0$ case, while the maximum ${\overline{w^{2}}}^{x 1 / 2}$ is about $50 \%$ larger in Fig. 9b than in Fig. 9a.

Figures $9 \mathrm{c}$ and $9 \mathrm{~d}$ show $\overline{w^{12}} x^{x / 2}$ much later in the simulations, at $t=18$ days. By this point, the degree of crossfrontal confinement is much more pronounced, with the $\Delta U_{\mathrm{BT}}=0.6 \mathrm{~m} \mathrm{~s}^{-1}$ case having ${\overline{w^{12}}}^{x 1 / 2}$ confined between $y=15$ and $35 \mathrm{~km}$, while, in the case with $\Delta U_{\mathrm{BT}}=0$, $\frac{w^{\prime 2}}{x 1 / 2}$ has stretched to fill almost the entire width of the domain.

The horizontally averaged alongfront velocity $\bar{u}^{x z}$ shows evidence of jet intensification at 18 days in the $\Delta U_{\mathrm{BT}}=0.6 \mathrm{~m} \mathrm{~s}^{-1}$ case. Figure 10a demonstrates that the jet magnitude has increased at the center of the front, $y=25 \mathrm{~km}$, while decreasing somewhat at the flanks. The barotropic velocity at the center of the front has increased by about $7 \%$ compared to the initial conditions. The cumulative result of these areas of jet weakening and strengthening is a sharpening of the jet; that is, the absolute magnitude of barotropic shear has increased 
(a)

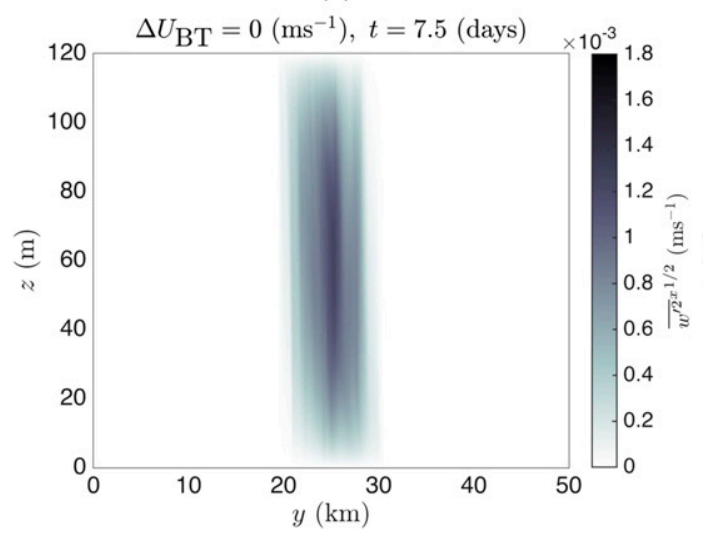

(c)

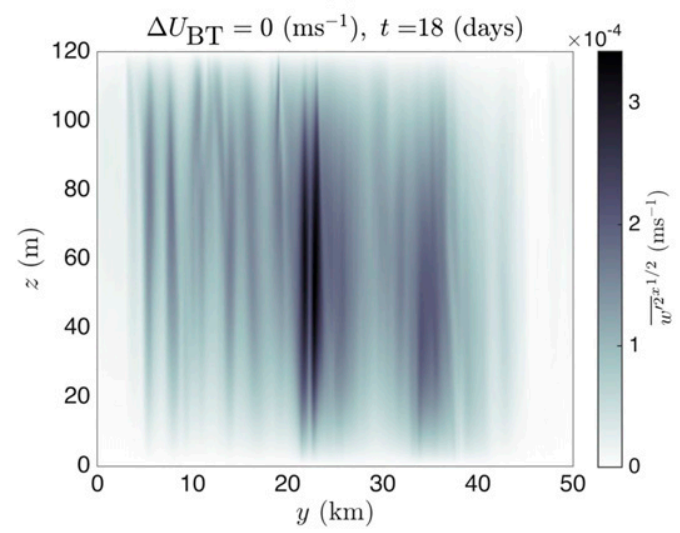

(b)

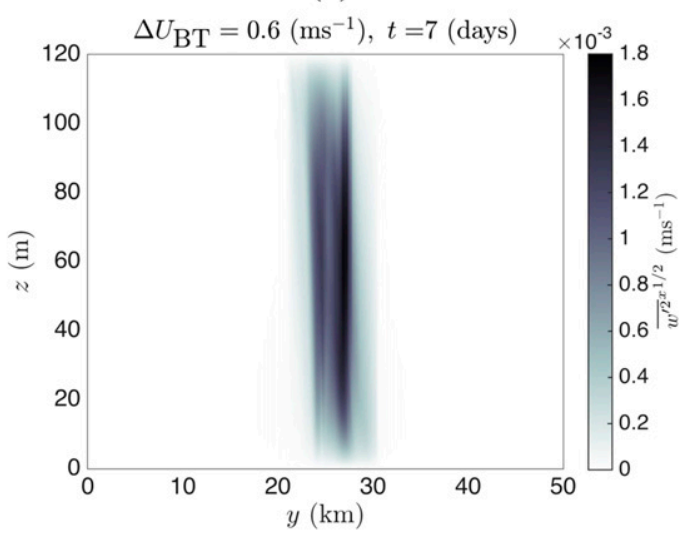

(d)

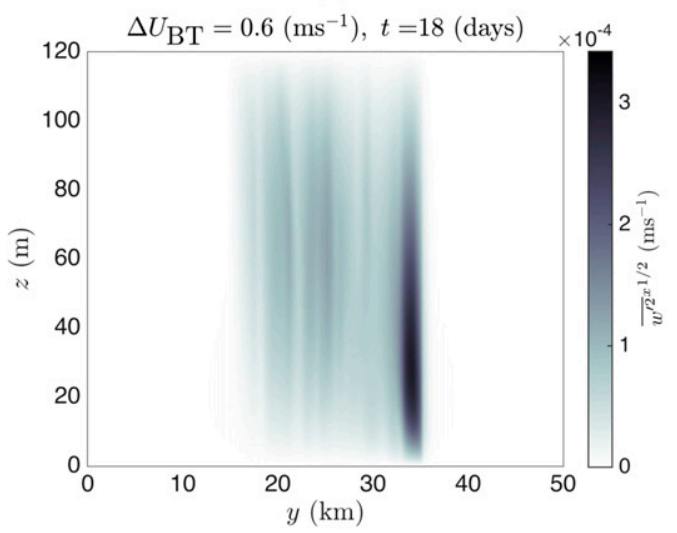

FIG. 9. Alongfront averaged rms vertical velocity ${\overline{w^{\prime 2}}}^{x 1 / 2}$ for (a),(c) $\Delta U_{\mathrm{BT}}=0$ and (b),(d) $\Delta U_{\mathrm{BT}}=0.6 \mathrm{~m} \mathrm{~s}^{-1}$. The time corresponds to the time of the second local maxima in domain-averaged rms vertical velocity (see Fig. 3b), and (c),(d) the time is 18 days.

between $y \approx 20$ and $30 \mathrm{~km}$ (see the red shaded portion of Fig. 10b).

This increase in shear is crucial in explaining the halting of baroclinic growth; increased shear near the front implies that BCI will be more influenced by the jet, with instabilities tending to be further deformed and tilted by the shear. These tilted modes will be prevented from attaining the same structure as the fastest-growing mode that would be present in the absence of strong shear. The intensification of the jet could help explain why, during the late stages of the $\Delta U_{\mathrm{BT}}=0.6 \mathrm{~m} \mathrm{~s}^{-1}$ simulation, we see stabilization of BCI and the crossfrontal confinement of baroclinic modes.

Another mechanism to describe jet strengthening is the cross-frontal horizontal shear production $\left(\mathrm{HSP}_{x} \equiv\right.$ $\left.-\overline{u^{\prime} v^{\prime}} \partial \bar{u}^{x} / \partial y\right)$, a term resulting from the eddy kinetic energy budget with Reynolds averaging applied in the $x$ direction only. The depth-averaged HSP at the time when growth of $\overline{\mathrm{EKE}}^{x y z}$ appears to saturate in the $\Delta U_{\mathrm{BT}}=0.6 \mathrm{~m} \mathrm{~s}^{-1}$ case, $t=7.5$ days, is shown in Fig. 11 . We see that the minimum in ${\overline{\mathrm{HSP}_{x}}}^{2}$ increases in magnitude with increasing jet strength $\Delta U_{\mathrm{BT}}$ and is focused on the center of the front. The connection between HSP and jet strength will be expanded upon in the following section.

\section{Linear stability analysis}

Here, we analyze the linear stability of the initial conditions described above. This is done by time stepping the nonhydrostatic Boussinesq equations, linearized about a basic state with arbitrary $y-z$ dependence. Perturbations to the basic state are expanded using a Fourier transform in $x$ :

$$
\left(u^{\prime}, v^{\prime}, w^{\prime}, b^{\prime}, \phi^{\prime}\right)=\operatorname{Re}\left[(\hat{u}, \hat{v}, \hat{w}, \hat{b}, \hat{\phi}) e^{i k x}\right]
$$

where $k$ is a prescribed wavenumber in the $x$ direction and variables denoted with a hat are functions of $y, z$, and $t$. At $t=0$, the variables denoted with a hat are initialized with small-amplitude random noise of the form: 
(a)

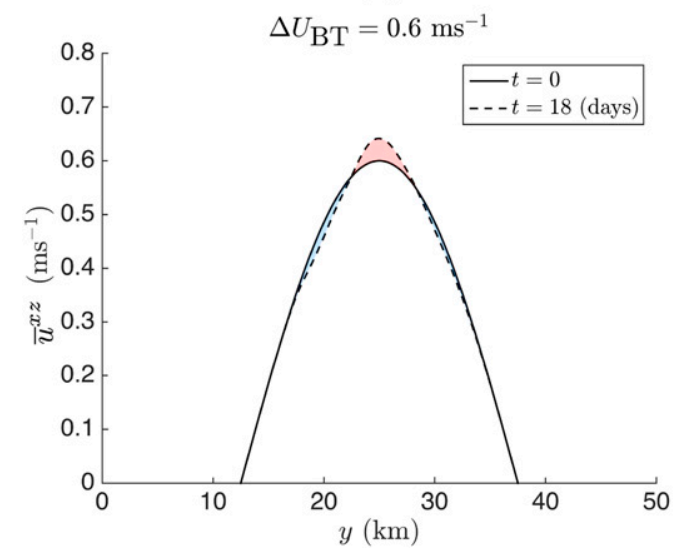

(b)

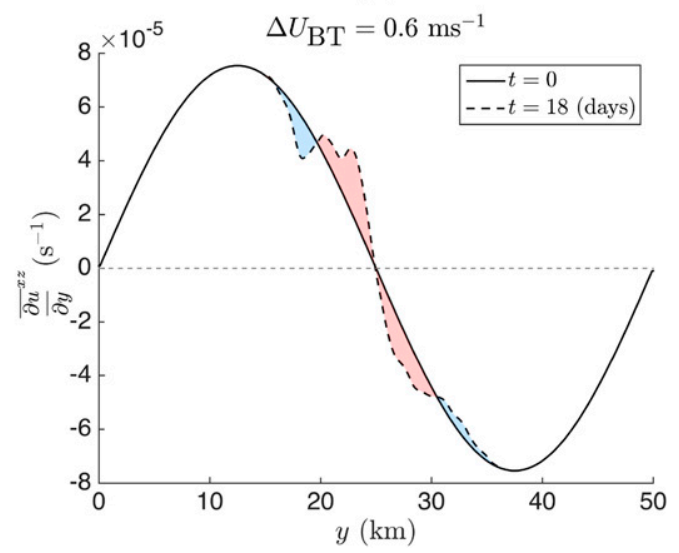

FIG. 10. (a) Depth- and alongfront-averaged velocity $\bar{u}^{x z}$ and (b) depth- and alongfront-averaged horizontal shear $\overline{\partial u}_{\partial y}{ }^{x z}$. Solid black lines show the values at $t=0$, that is, the initial barotropic jet $u_{\mathrm{BT}}$ and its corresponding shear. Dashed lines show the same quantities at $t=18$ days. Red and blue shading highlight regions where the velocity and shear have increased or decreased in amplitude, respectively.

$$
\hat{u}(y, z, t=0)=A \sum_{k} \sum_{m} e^{i l y+i m z+\phi}, \quad \text { etc. }
$$

where $A$ is an arbitrary complex amplitude and $\varphi$ is a random phase shift. For each wavenumber $k$, we then time step the linearized governing equations, neglecting any nonlinear terms of the form $a^{\prime} b^{\prime}$, where primes denote perturbations from the initial conditions, until they converge to the fastest-growing mode for each wavenumber. Specifically, we time step the linearized equations until the growth rate, given by

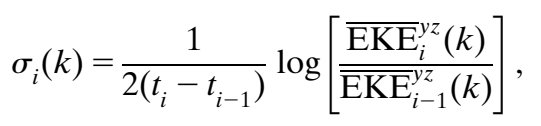

is approximately constant in time, where $i$ denotes the time step. For each time step, we calculate the mean and standard deviation of the growth rates $\sigma_{i}$ over the past $N_{C}$ time steps. For a chosen number of time steps $N_{C}$ and convergence threshold $\delta_{C}$, we determine that the growth rate has converged at time step $i$ and wavenumber $k$ if

$$
\frac{\sqrt{\frac{1}{N_{C}} \sum_{j=i-N_{C}}^{i}\left|\sigma_{j}(k)-\frac{1}{N_{C}} \sum_{m=i-N_{C}}^{i} \sigma_{m}(k)\right|^{2}}}{\frac{1}{N_{C}} \sum_{n=i-N_{C}}^{i} \sigma_{n}(k)}<\delta_{C},
$$

where $\delta_{C}$ is a small parameter. In other words, we require that the standard deviation is no more than $\delta_{C}$ times larger than the mean growth rate over the last $N_{C}$ time steps.

All parameters are kept the same as described in section 3a, except $N^{2}$. Having an analytic form for SI in the inviscid case and noting that the growth rate of SI was nearly identical across all simulations, we are instead interested in predicting BCI growth rates. With this in mind, we take the initial stratification to be $N^{2}=3 \times 10^{-6} \mathrm{~s}^{-2}$ such that $f q \geq 0$ everywhere in the domain, ensuring stability with regard to SI. Note that the basic state does not include the inertial oscillations that appear in the simulations after the SI phase.

The viscosity and diffusivity applied to the perturbations match those used in the numerical simulations; specifically, $\nu_{H}=1 \mathrm{~m}^{2} \mathrm{~s}^{-1}, \nu_{V}=5 \times 10^{-5} \mathrm{~m}^{2} \mathrm{~s}^{-1}$, and $\operatorname{Pr}=\nu / \kappa=1$. Here the number of grid points is $N_{y}=150$ and $N_{z}=50$ in the $y$ and $z$ directions, respectively. We use a fixed time step of $150 \mathrm{~s}$. The time-averaging interval required for achieving convergence is chosen to

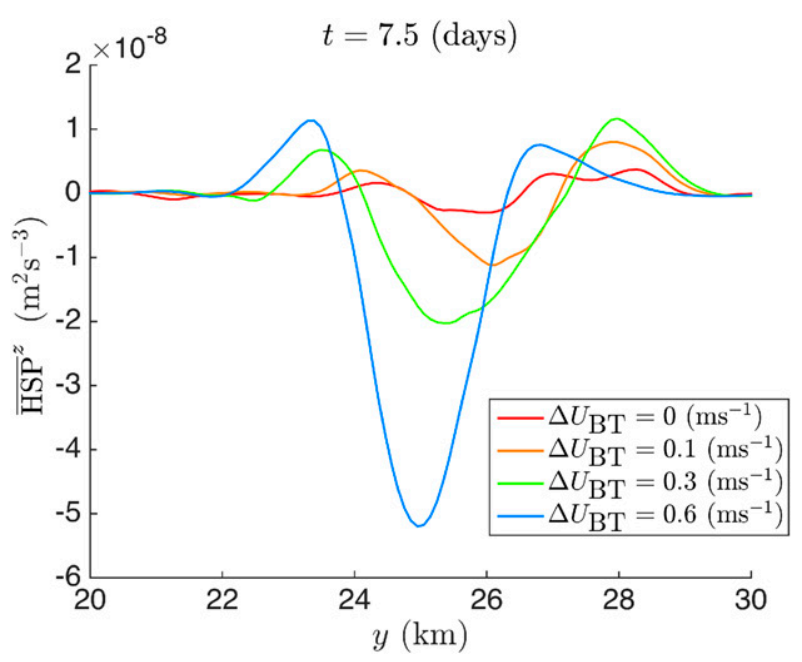

FIG. 11. Depth-averaged horizontal shear production $\left(\overline{\mathrm{HSP}}^{z}\right)$ at 7.5 days for each barotropic jet strength. 
be 10 days $\left(N_{C}=5760\right)$, with the growth rate tolerance chosen to be $1 \%$ of the standard deviation of the growth rate; that is, $\delta_{C}=0.01$. Although the time required to reach a converged state varies from one case to another, in all cases the growth rate achieved the demanded tolerance over the 10-day averaging window before $t=$ 70 days.

Figure 12 shows the growth rate associated with the most unstable modes for barotropic jets strengths $\Delta U_{\mathrm{BT}}=0,0.1,0.2,0.3,0.4,0.5$, and $0.6 \mathrm{~m} \mathrm{~s}^{-1}$. For $\Delta U_{\mathrm{BT}}=0$, the maximum growth rate occurs for a wavelength $\lambda=2 \pi k=9 \mathrm{~km}$. For the next two increases in barotropic jet strength, $\Delta U_{\mathrm{BT}}=0.1$ and $0.2 \mathrm{~m} \mathrm{~s}^{-1}$, the maximum growth rate decreases and the overall growth rate curve flattens. This trend reverses for further increases in $\Delta U_{\mathrm{BT}}$, with the maximum growth rate once again increasing. However, for the barotropic jet strengths considered, the maximum growth rate never quite recovers to that for the simulation with no barotropic jet added $\left(\Delta U_{\mathrm{BT}}=0\right)$. The maximum growth rate with $\Delta U_{\mathrm{BT}}=0.6 \mathrm{~m} \mathrm{~s}^{-1}$ is $10 \%$ lower than that with $\Delta U_{\mathrm{BT}}=0 \mathrm{~m} \mathrm{~s}^{-1}$.

The dependence of the growth rate on the barotropic jet is qualitatively different than what was reported in James (1987) and Nakamura (1993a) [e.g., see Fig. 5 of James (1987)]. They considered constant barotropic shear and observed a monotonic reduction in maximum growth rate with increasing barotropic shear. In addition, they reported a shift of the growth rate maximum to larger alongfront wavelengths with increased barotropic shear. While this indeed appears to be the case for the first two jet strengths $\Delta U_{\mathrm{BT}}=0.1$ and $0.2 \mathrm{~m} \mathrm{~s}^{-1}$, these trends reverse for the higher jet strengths considered here. Unlike James (1987) and Nakamura (1993a), the imposed barotropic jet in our case has nonconstant shear and associated variations in potential vorticity gradients. As will be shown below, these have competing influences on submesoscale BCI.

To see the influence of horizontal shear on BCI, it is illustrative to look at the structure of the fastest-growing modes. Figure 13 shows contours of buoyancy perturbations with $\lambda=9 \mathrm{~km}$ from the cases with $\Delta U_{\mathrm{BT}}=0$ and $0.6 \mathrm{~m} \mathrm{~s}^{-1}$ at the top of the domain $(z=120 \mathrm{~m})$. These contours show that baroclinic modes are centered on the cold side of the front, with $y<25 \mathrm{~km}$, and form a boomerang-like shape. In the $\Delta U_{\mathrm{BT}}=0$ case the boomerang shape is less prominent, caused only by the horizontal shear arising from the thermal wind. The boomerang shape of the modes is more pronounced when $\Delta U_{\mathrm{BT}}=0.6 \mathrm{~m} \mathrm{~s}^{-1}$ at the top surface of the domain where the additional shear from the barotropic jet further deforms the baroclinic modes.

The deformed baroclinic modes have a significant effect on the cross-front momentum flux. The deformation

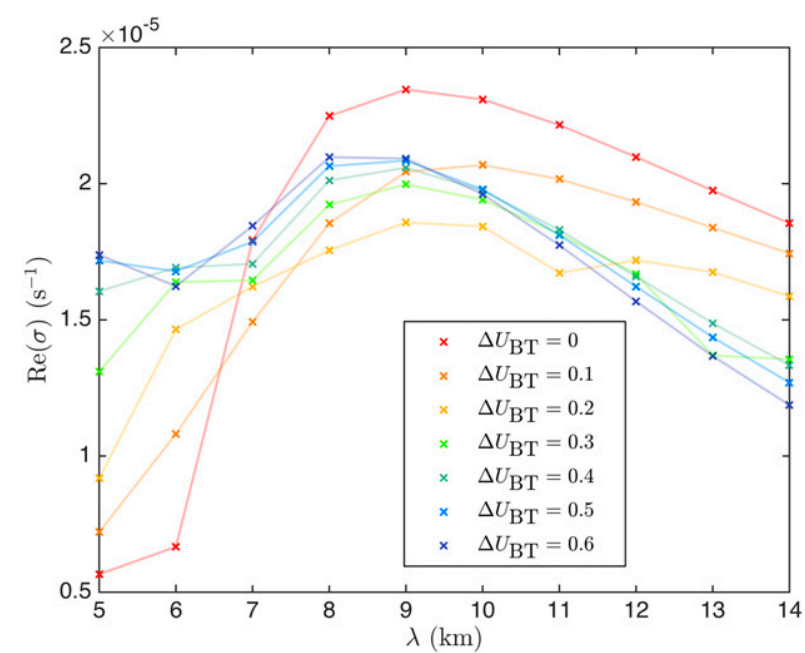

FIG. 12. Growth rate $\sigma$ of the most unstable mode from a linear stability analysis, plotted as a function of alongfront wavelength, $\lambda=2 \pi / k$, for various barotropic jet amplitudes, $\Delta U_{\mathrm{BT}}=0,0.1,0.2$, $0.3,0.4,0.5$, and $0.6 \mathrm{~m} \mathrm{~s}^{-1}$. The growth rates were calculated for wavelengths between 5 and $14 \mathrm{~km}$, with a 1-km step. Each calculated growth rate is indicated by a cross, with lines plotted to guide the eye only.

of the baroclinic mode into a rightward-oriented boomerang, as seen in Fig. 13b, results in negative cross-front momentum fluxes, $\frac{u^{\prime} v^{\prime}}{x}<0$, on the warm side of the front $(y>25 \mathrm{~km})$ and positive cross-front momentum fluxes, ${\overline{u^{\prime} v^{\prime}}}^{x}>0$, on the cold side of the front $(y<25 \mathrm{~km})$. The net result is a convergence of cross-frontal, horizontal momentum toward the center of the front at $y=25 \mathrm{~km}$. This convergence of momentum results in decreased horizontal shear production associated with the barotropic jet, given by HSP $\equiv-{\overline{u^{\prime} v^{x}}}^{x} d{\overline{u_{\mathrm{BT}}}}^{x} / d y$, illustrated by the barotropic jet strengthening seen in the simulation with $\Delta U_{\mathrm{BT}}=0.6 \mathrm{~m} \mathrm{~s}^{-1}$ (see Fig. 10). This is the same mechanism of momentum transfer as that induced by "banana shaped" eddies, which are known to be responsible for meridional transfer of momentum at synoptic scales in the atmosphere [as discussed, e.g., by Marshall and Plumb (2008); see their Fig. 8.14].

Figure 14a shows, for each barotropic jet strength, with $\lambda=9 \mathrm{~km}$, a decomposition of the eddy kinetic energy budget associated with the most unstable modes into the three most significant contributions: the buoyancy flux $\overline{b^{\prime} w^{\prime}} x z$ (green), the horizontal (barotropic) shear production (blue), and the geostrophic shear production, GSP $\equiv-\overline{u^{\prime} w^{\prime}} d \bar{u} x / d z$ (orange). Each term has been normalized by the mean eddy kinetic energy $\left(\overline{\mathrm{EKE}}^{x z}\right)$. From $\Delta U_{\mathrm{BT}}=0$ to $0.2 \mathrm{~m} \mathrm{~s}^{-1}$ we see that the GSP increases, while the HSP and buoyancy fluxes decrease. Negative HSP indicates a transfer of energy from eddy kinetic energy to the kinetic energy associated with the 
(a)

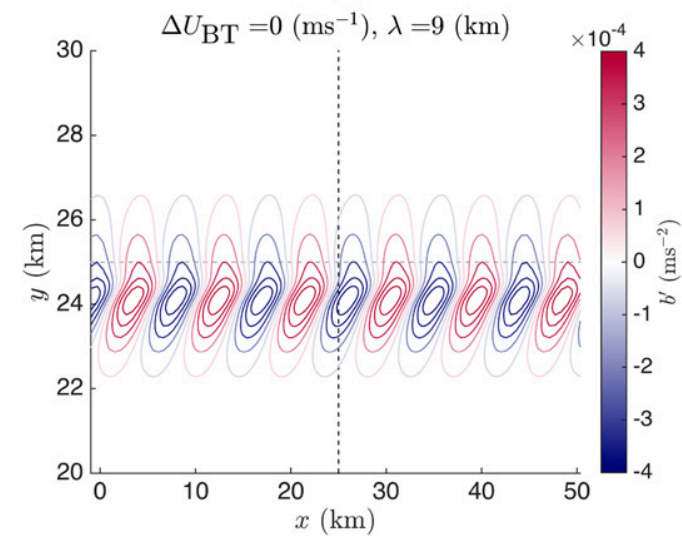

(b)

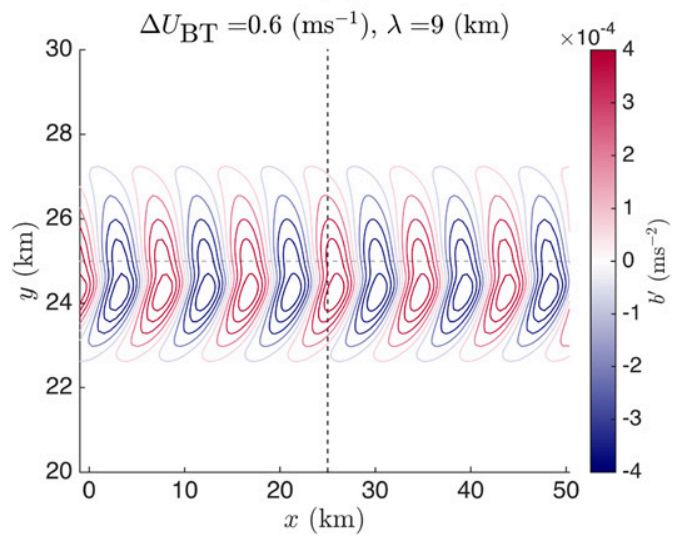

FIG. 13. Horizontal structure of buoyancy perturbations for the fastest-growing mode with $\lambda=9 \mathrm{~km}$ for (a) $\Delta U_{\mathrm{BT}}=0$ and (b) $\Delta U_{\mathrm{BT}}=0.6 \mathrm{~m} \mathrm{~s}^{-1}$ at the top surface $(z=120 \mathrm{~m})$.

barotropic jet. This pathway becomes more effective with increased barotropic jet strength (up to $\Delta U_{\mathrm{BT}}=$ $0.2 \mathrm{~m} \mathrm{~s}^{-1}$ ), while the changes in buoyancy fluxes and GSP approximately cancel one another out.

For further increases in barotropic jet strength, $\Delta U_{\mathrm{BT}}>0.2 \mathrm{~m} \mathrm{~s}^{-1}$, the trends in geostrophic shear production and buoyancy fluxes reverse. The buoyancy fluxes increase more rapidly than the geostrophic shear production decreases. Horizontal shear production stays approximately constant for further increases in jet strength, $\Delta U_{\mathrm{BT}}>0.2 \mathrm{~m} \mathrm{~s}^{-1}$. Overall the increase in growth rate for increasing jet strength $\Delta U_{\mathrm{BT}}$ appears to be driven predominantly by increases in the buoyancy flux.

(a)

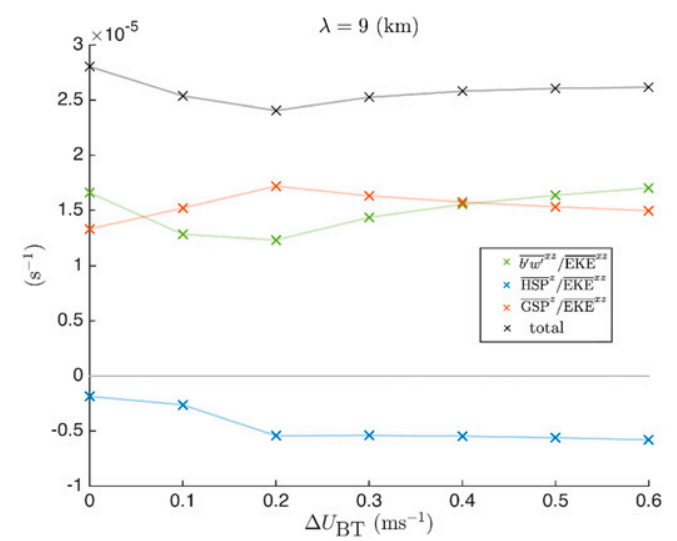

\section{a. Effective $\beta$}

Our aim in this subsection is to isolate the effect of the potential vorticity gradient associated with the horizontally sheared barotropic jet from the horizontal shear production. To do this, we remove explicit advection associated with the barotropic jet but retain its influence on the potential vorticity by modifying the Coriolis parameter such that

$$
f=f_{0}+\int_{L_{y} / 2}^{y} \beta_{\mathrm{eff}} d y^{\prime}
$$

where $f_{0}$ is the usual $f$-plane Coriolis parameter and

\section{(b)}

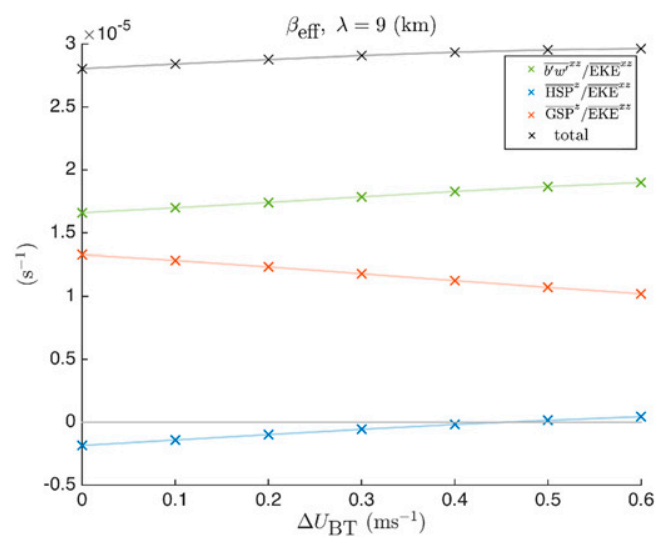

FIG. 14. Dominant source terms in the kinetic energy budget from the linear stability analysis. Crosses indicate the growth rate for $\lambda=9 \mathrm{~km}$ from the three dominant contributions to EKE growth: 1 ) buoyancy flux $\overline{b^{\prime} w^{\prime x}}$ (green), 2) horizontal (barotropic) shear production $\left(\overline{\mathrm{HSP}^{z}}\right)($ blue $)$, and 3) vertical (geostrophic) shear production $\left(\overline{\mathrm{GSP}}^{z}\right)$. Black crosses indicate the sum of these three contributions. All terms have been divided by the mean EKE $\left(\overline{\mathrm{EKE}}^{x z}\right)$. Shown are (a) full barotropic jet cases and (b) $\beta_{\text {eff }}$ cases. 


$$
\beta_{\mathrm{eff}}=-\frac{d^{2} u_{\mathrm{BT}}}{d y^{2}}
$$

With $u_{\mathrm{BT}}$ given by the cosine jet above [Eq. (3)], $f$ is

$$
f=f_{0}-\frac{d u_{\mathrm{BT}}}{d y} .
$$

We note that potential vorticity of the original initial conditions, with $f=f_{0}$ and $u=u_{W}+u_{\mathrm{BT}}$, can be written as

$$
\begin{aligned}
q & =\left(f_{0} \mathbf{k}+\nabla \times \mathbf{u}\right) \cdot \nabla b \\
& =\left[\left(f_{0}-\frac{d u_{\mathrm{BT}}}{d y}\right) \mathbf{k}+\nabla \times\left(\mathbf{u}-u_{\mathrm{BT}} \mathbf{i}\right)\right] \cdot \nabla b .
\end{aligned}
$$

If we instead take $f=f_{0}-d u_{\mathrm{BT}} / d y$ and $u=u_{W}$, that is, with the barotropic jet absent from the initial velocity field but an additional $\beta_{\text {eff }}$ term included in $f$, then potential vorticity associated with the initial conditions remains exactly as in Eq. (16). These new initial conditions and modified Coriolis parameter $f$ then allow us to capture the contribution to the potential vorticity from the barotropic jet while eliminating advection and horizontal shear production associated with the barotropic jet.

Note that our approach is different from simply removing the advection terms involving the operator $\mathbf{u}_{\mathrm{BT}} \cdot \nabla$ from the momentum equations. Doing so would leave a term $v d u_{\mathrm{BT}} / d y$ in the $x$-momentum equation while $u_{\mathrm{BT}}$ would not appear in the $y$-momentum equation. This choice would result in a jet able to transfer energy to and from the growing perturbations through horizontal shear production. Instead, our approach effectively adds an extra term, $-u d u_{\mathrm{BT}} / d y$, to the $y$ momentum equation. While arguably less physical, this approach eliminates the shear production term associated with the barotropic jet from the perturbation energy budget. As a result, $u_{\mathrm{BT}}$ does not appear in the perturbation energy equation, and instead the perturbations are modified by the same potential vorticity gradient that would be induced by the barotropic jet.

We repeat the linear stability analysis described above with this new initial velocity profile, $u=u_{W}$, and additional $\beta_{\text {eff }}$ term added to the Coriolis parameter $f$. We vary the magnitude of $\beta_{\text {eff }}$ by matching to the PV effect of $\Delta U_{\mathrm{BT}}=0,0.1,0.2,0.3,0.4,0.5$, or $0.6 \mathrm{~m} \mathrm{~s}^{-1}$. Figure 15 shows the resulting growth rate for each magnitude of $\beta_{\text {eff }}$. In contrast to the full barotropic jet cases, we now see a monotonic increase in maximum growth rate as we increase $\Delta U_{\mathrm{BT}}$. The $\Delta U_{\mathrm{BT}}=0.6 \mathrm{~m} \mathrm{~s}^{-1}$ case has a growth rate $12 \%$ higher than the $\Delta U_{\mathrm{BT}}=0 \mathrm{~m} \mathrm{~s}^{-1}$ case. There is also a shift to smaller wavelengths as we increase $\Delta U_{\mathrm{BT}}$,

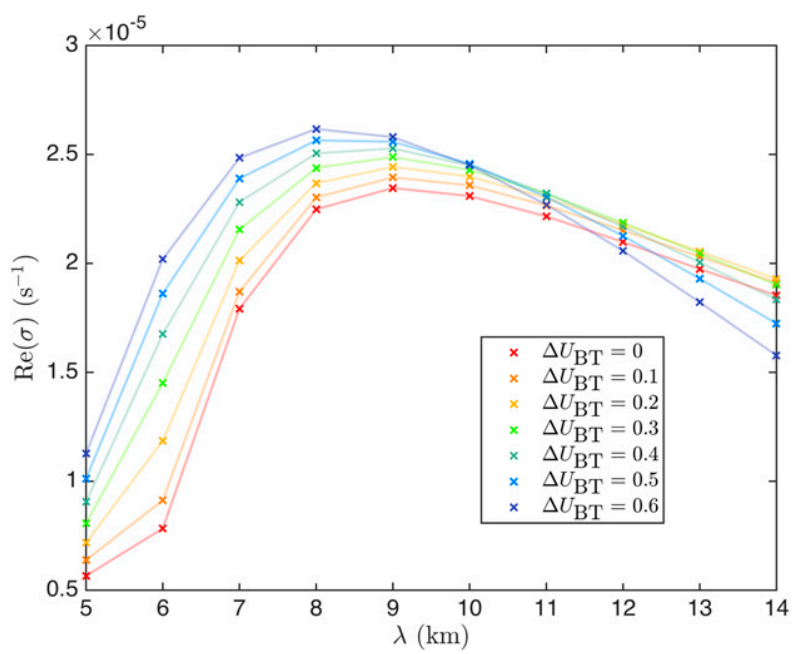

FIG. 15. Growth rate $\sigma$ of the most unstable mode as a function of alongfront wavelength, $\lambda=2 \pi / k$, for the linear stability analysis with $\beta_{\text {eff }}$ and $\Delta U_{\mathrm{BT}}=0,0.1,0.2,0.3,0.4,0.5$, and $0.6 \mathrm{~m} \mathrm{~s}^{-1}$. Growth rates were calculated for wavelengths between 4 and $16 \mathrm{~km}$, with a 1-km step, and each computed solution is indicated by a cross. Lines plotted are to guide the eye only.

with the fastest-growing wavelength moving from $\lambda=$ $9 \mathrm{~km}$ for $\Delta U_{\mathrm{BT}}=0$ to $\lambda=8 \mathrm{~km}$ for $\Delta U_{\mathrm{BT}}=0.6 \mathrm{~m} \mathrm{~s}^{-1}$.

Figure $14 \mathrm{~b}$ shows the same energy budget as Fig. 14a but now with $\beta_{\text {eff }}$ replacing the barotropic jet. The trends in each of these terms are now monotonic as $\Delta U_{\mathrm{BT}}$ increases. Buoyancy fluxes and horizontal shear production increase as $\Delta U_{\mathrm{BT}}$ increases, while geostrophic shear production decreases. It is unclear what it is, intrinsically, about the inclusion of the effective $\beta$ term that drives the increase in growth rate. Since our $\beta_{\text {eff }}$ approach has the effect of modifying the potential vorticity while eliminating the horizontal shear production associated with the barotropic jet, the distribution of potential vorticity appears to play an important role.

Figure 16 compares the maximum growth rates between two sets of linear stability calculations: with a barotropic jet (orange crosses) and with an effective $\beta$ term (blue crosses). For comparison, the maximum growth rate without a barotropic jet and with a constant Coriolis parameter is indicated with a dashed line. When an effective $\beta$ term is present, the maximum growth rate increases with increasing $\Delta U_{\mathrm{BT}}$, while the maximum growth rate decreases with $\Delta U_{\mathrm{BT}}$ when accompanied by a barotropic jet. This result implies that the effects of barotropic shear and PV gradient sign changes associated with a barotropic jet oppose one another, with increased barotropic shear resulting in decreased growth rates while modulations of the PV gradient associated with $\beta_{\text {eff }}$ increase growth rates. There is some evidence that the reduction in growth rate in the case with a 


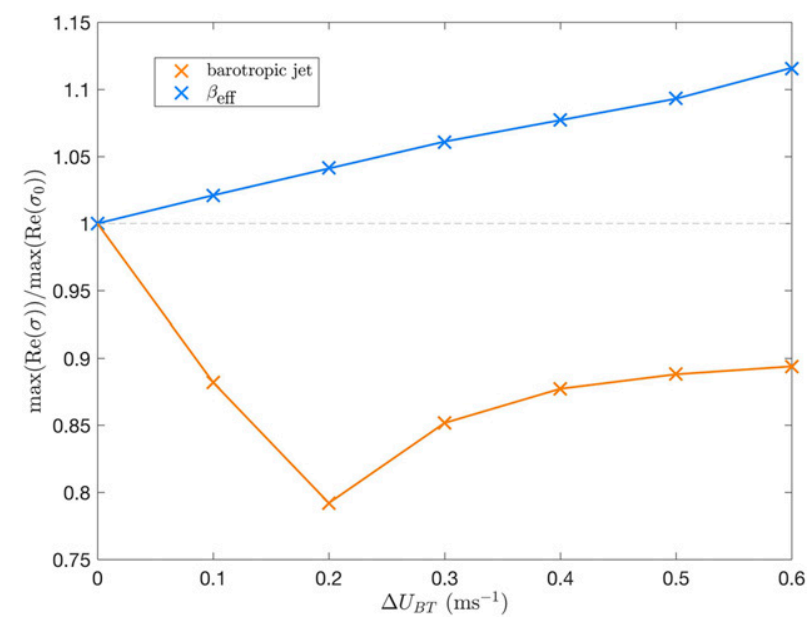

FIG. 16. Maximum growth rate of the most unstable modes with wavelengths $\lambda=4-16 \mathrm{~km}$ normalized by the maximum growth rate without a barotropic jet. Orange and blue crosses indicate cases with a barotropic jet and with $\beta_{\text {eff }}$, respectively. The dashed line indicates the normalized growth rate without a barotropic jet for comparison.

barotropic jet saturates for $\Delta U_{\mathrm{BT}}>0.4 \mathrm{~m} \mathrm{~s}^{-1}$, while the maximum growth rate continues to increase as a function of $\Delta U_{\mathrm{BT}}$ with $\beta_{\mathrm{eff}}$.

\section{b. Linear stability of final state}

As seen in Fig. 16, the addition of a barotropic jet reduces the maximum growth rate by about $20 \%$ at most. This suggests that the saturation and confinement of submesoscale disturbances in the simulations with a strong barotropic jet cannot be explained by the barotropic governor acting on small-amplitude perturbations to the initial conditions. To analyze the influence of the barotropic jet on the stability of the front at the end of the numerical simulations, we repeated the linear stability analysis with initial conditions formed by averaging the final state from the simulation with $\Delta U_{\mathrm{BT}}=0.6 \mathrm{~m} \mathrm{~s}^{-1}$ in the alongfront $(x)$ direction. Viscosity, spatial resolution, time step, and convergence parameters were the same as described in section 4.

Three variations of the linear stability analysis were performed. The first case uses a basic state consisting of the $x$-averaged buoyancy from the simulation with the idealized thermal wind and barotropic velocity components as in Eqs. (2) and (3) (labeled "idealized $u$ "). In the second case, the basic state consists of the $x$-averaged velocity and buoyancy from the end of the numerical simulations (labeled "computed $u$ "). Finally, the third case has a basic state consisting of the $x$-averaged barotropic velocity and buoyancy from the numerical simulations, but with an idealized baroclinic component of the velocity as in Eq. (2) [labeled "computed $u_{\mathrm{BT}}$ (balanced)"]. For comparison, the maximum growth rate associated with the initial conditions is shown as a red curve, which is positive (unstable) for all wavelengths shown. In contrast, the idealized $u$ case shows positive growth only for wavelengths $\lambda=9-14 \mathrm{~km}$ (orange), while the last two cases do not have any growing modes for the wavelengths considered. The green curve confirms that the basic state consisting of $x$-averaged buoyancy and velocity from the end of the simulation with $\Delta U_{\mathrm{BT}}=0.6 \mathrm{~m} \mathrm{~s}^{-1}$ is indeed stable. Further, the blue curve indicates that stabilization of the basic state can be achieved without modification of the initial baroclinic velocity.

The difference between the computed $u$ and idealized $u$ cases is particularly interesting. The fact that growth rates have been vastly reduced in the idealized $u$ case (orange) when compared with growth rates from the initial conditions (red) indicates that changes to the mean buoyancy, including variations in the structure of the front (including frontal strength and restratification), have a substantial impact on the linear stability of the flow. Further, using the $x$-averaged velocity field from the numerical simulation reduces growth rates (green), indicating that the full stability of the flow is sensitive to these modest changes in the velocity field, including strengthening of the barotropic jet.

Figure 17 shows the decomposition of the growth rate into contributions from the buoyancy flux, geostrophic shear production, and barotropic shear production terms as for Fig. 14. The three panels indicate results from the initial conditions (left), computed $b$ and idealized $u$ (center), and computed $b$ and $u$ (right). We see that the geostrophic shear production and buoyancy flux are vastly reduced between the left and center panels, consistent with reduced growth rates of BCI. An evaluation of the Charney-Stern-Pedlosky stability criteria indicates that the necessary, though not sufficient, conditions for instability are always satisfied in all cases shown in Fig. 18, although it is apparent that both computed $u$ cases are in fact (marginally) stable. The right panel of Fig. 17 indicates that all energetic pathways have been effectively shut down in this late stage of the simulation, with each term now approximately zero.

\section{Summary and conclusions}

Motivated by observations of a front in the Southern Ocean, this paper presents the nonlinear evolution of submesoscale instability at an isolated front with a collocated barotropic jet of varying amplitude. Beginning with an unstratified mixed layer, $N^{2}=0$, the initial conditions chosen were unstable to both SI and BCI. We find SI growth rates similar to those predicted with 

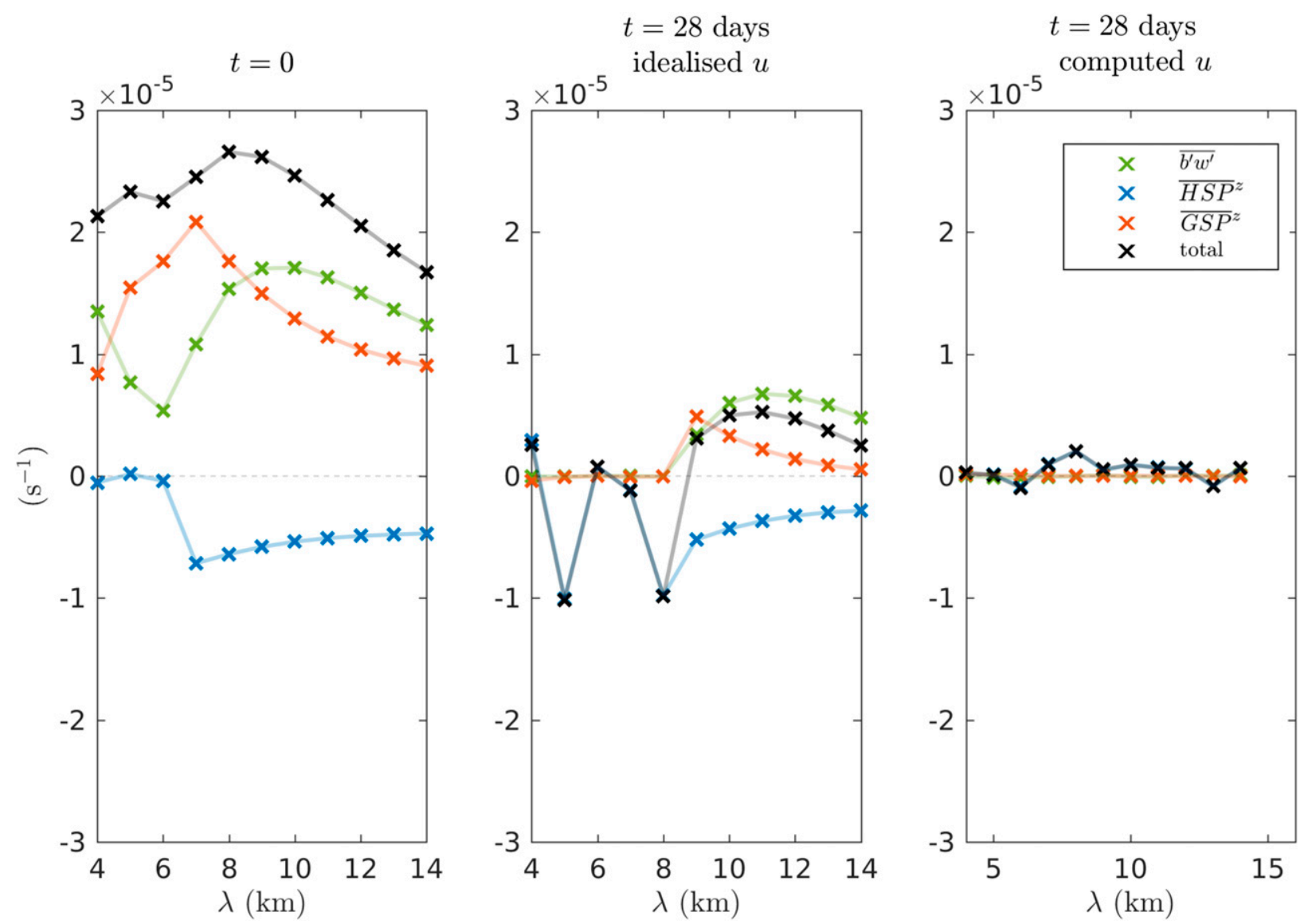

FIG. 17. Decomposition of the growth rate based on the terms in the perturbation energy budget based on a linear stability analysis. All terms are normalized by the perturbation kinetic energy. Results from using (left) the idealized initial conditions and basic state with the buoyancy field constructed by applying an $x$ average to the numerical simulation with $\Delta U_{\mathrm{BT}}=0.6 \mathrm{~m} \mathrm{~s}^{-1}$ at $t=28$ days with the velocity based on the (center) idealized $u$ and (right) model $u$ as described in the text. Crosses indicate the proportion of EKE growth from three

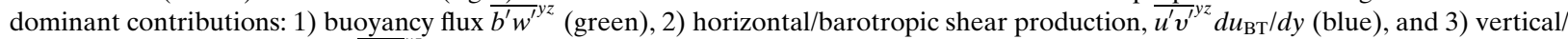
geostrophic shear production, $\bar{u}^{\prime} w^{\prime y z} d \bar{u}_{W}^{y z} / d z$ (orange). Black crosses indicate the sum of these three components. Lines are to guide the eye only.

$\mathrm{Ri}=0.25$ and interpret this as being due to an initial adjustment toward $\mathrm{Ri}=0.25$ caused by small-scale instability resulting from the initial small-amplitude random noise added to the initial conditions. Though, in a domain-averaged sense, SI growth rates are similar for each barotropic jet strength, SI has higher growth rates on the warm side of the front, particularly for higher barotropic jet strength. This reflects the larger linear growth rate predicted for SI in regions of strong anticyclonic barotropic relative vorticity. As in Stamper and Taylor (2017), steps form in the cross-front surface buoyancy profile near the center of the front.

BCI begins at approximately the same time for each barotropic jet strength. However, as time evolves, the eddy kinetic energy continues to grow in the case with no barotropic jet $\left(\Delta U_{\mathrm{BT}}=0\right)$, while it decays at late times in the case with strongest barotropic jet $\left(\Delta U_{\mathrm{BT}}=0.6 \mathrm{~m} \mathrm{~s}^{-1}\right)$.
In the case with the strongest jet (representing the closest match with the barotropic jet observed during the SMILES cruise), the final state retains a sharp front where the buoyancy perturbations and large rms vertical velocity are confined. This contrasts strongly with the case with no barotropic jet, in which strong baroclinic eddies persist at late times and propagate to the domain boundaries. Thus, the addition of a strong barotropic jet allows for the equilibration of submesoscale disturbances at the front.

In cases with a barotropic jet, during the early stages of $\mathrm{BCI}$, there is pronounced negative horizontal shear production (HSP) near the center of the front. HSP increases in magnitude with increasing barotropic jet strength. Such negative horizontal shear production, associated with the flux of kinetic energy from the perturbations to the barotropic jet, coincides with the 


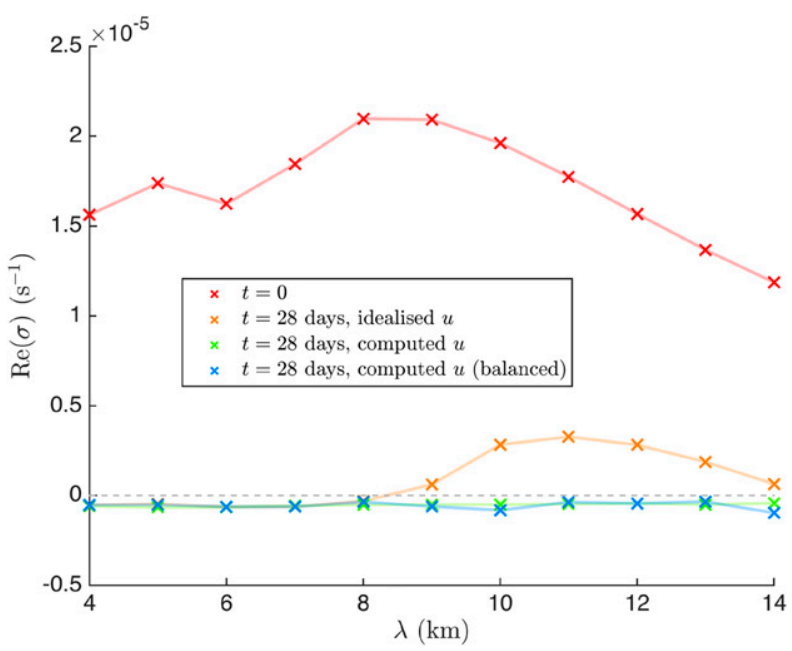

FIG. 18. Growth rate of the most unstable mode for $\Delta U_{\mathrm{BT}}=$ $0.6 \mathrm{~m} \mathrm{~s}^{-1}$ with the initial conditions (red) and the final state of the simulation at $t=28$ days with computed buoyancy $b$ and idealized alongfront velocity $u$, as in Eqs. (2) and (3) (orange); computed buoyancy $b$ and computed velocity $u$ (green); and computed buoyancy $b$ and computed barotropic component of the alongfront velocity $u$ but with an idealized thermal wind component as in Eq. (2) (blue).

strengthening of the barotropic jet and barotropic shear for the largest initial barotropic jet strength, $\Delta U_{\mathrm{BT}}=0.6 \mathrm{~m} \mathrm{~s}^{-1}$.

To gain a broader understanding of the influence of the barotropic jet, we conducted a linear stability analysis of a barotropic jet superposed on an isolated front. The influence of the barotropic jet on the growth rate of the most unstable mode is modest. The maximum growth rate for the strongest barotropic jet strength considered, $\Delta U_{\mathrm{BT}}=0.6 \mathrm{~m} \mathrm{~s}^{-1}$, is $\sim 10 \%$ smaller than that for $\Delta U_{\mathrm{BT}}=0$. However, the maximum growth rate is a nonmonotonic function of the barotropic jet strength, initially decreasing for $\Delta U_{\mathrm{BT}}=0.1-0.2 \mathrm{~m} \mathrm{~s}^{-1}$ and increasing with subsequent increases in $\Delta U_{\mathrm{BT}}$. This result runs counter to work by James (1987) and Nakamura (1993a), which showed monotonic growth-rate changes with barotropic shear increases. One explanation for this difference is that our more complicated initial conditions introduce new physical processes to the problem.

To separate the influence of horizontal barotropic shear and potential vorticity (PV) gradients on the stability of the front, we analyzed the stability of initial conditions without an explicit barotropic jet but with an effective $\beta$ term added to the Coriolis parameter $f$, such that the PV was unchanged but the horizontal shear production associated with the barotropic jet was eliminated. In this case, increasing $\Delta U_{\mathrm{BT}}$ resulted in larger maximum growth rates. The linear stability analysis shows that the effects of variations in barotropic shear and potential vorticity gradients, resulting from the addition of a barotropic jet, oppose one another. An increase in the barotropic shear reduces the growth rate of BCI, as found by James (1987) and Nakamura (1993a), while changes to the PV gradient induced by the effective $\beta$ term result in increased $\mathrm{BCI}$ growth rates.

The linear stability analysis suggests that the barotropic governor is not sufficient to prevent submesoscale instabilities associated with the initial conditions. Another mechanism is needed to explain the apparent stabilization of the front at the end of the simulations with a strong barotropic jet. A linear stability analysis with a basic state consisting of the $x$-averaged buoyancy and alongfront velocity from the end of the simulations with $\Delta U_{\mathrm{BT}}=0.6 \mathrm{~m} \mathrm{~s}^{-1}$ shows that the mean flow is linearly stable. Tests using various combinations of initialand final-state flow variables show that the modification of the mean buoyancy and the strengthening of the barotropic jet are crucial to stabilizing the front in the simulations. This suggests that nonlinear processes are involved in the stabilization of the front.

This result qualitatively resembles the suppression of larger-scale turbulence in geostrophic turbulence (Rhines 1979; Vallis and Maltrud 1993). However, the problem studied here is a fully three-dimensional, nonhydrostatic, Boussinesq system and the evolution of the potential vorticity and stratification appear to be key to understanding the nonlinear equilibration of the front. In addition, the beta effect is not imposed externally by tangent plane rotation or topography but arrived at as a consequence of the resulting flow profile.

This paper joins other recent papers (Mahadevan et al. 2010; Fox-Kemper et al. 2011; Bachman and FoxKemper 2013; Ramachandran et al. 2014; Callies and Ferrari 2018a,b; Whitt and Taylor 2017) in clarifying how the long time evolution of BCI, both with and without winds and convection, differs from that arising from the Fox-Kemper et al. (2008) parameterization. That parameterization captures only the early-time behavior after BCI reaches finite amplitude, while the fronts themselves are resolved in the coarse model (roughly days 5-10 here). While this parameterization would therefore be expected to work well in the early stages of the flow evolution, the complications arising from inverse energy cascades, barotropic jet effects, coupling to mesoscale instabilities, and convective organization, for example, result in deviations at late times. Interestingly, the influence of the barotropic jet effects studied here appears to be the only case tending to stabilize BCI and reduce restratification, while the other studies find restratification rates enhanced in comparison to Fox-Kemper et al. (2008). A final linear stability analysis was undertaken with the buoyancy field 
and barotropic flow from the simulation corresponding to the largest barotropic jet strength but with flow in thermal wind balance. This configuration also resulted in a fully stabilized field. This was particularly interesting as it suggested that a geostrophically balanced version of the final state was linearly stable. This finding motivates future analysis regarding whether this process can be considered as a process of continual mixing and geostrophic adjustment of the flow.

Acknowledgments. M.S. and J.T. were funded through support from the Natural Environment Research Council, Award NE/J010472/1. B.F.-K. was supported by NSF 1350795 and a St. John's College Beaufort Visiting Scholarship.

\section{REFERENCES}

Bachman, S., and B. Fox-Kemper, 2013: Eddy parameterization challenge suite I: Eady spindown. Ocean Modell., 64, 12-28, https://doi.org/10.1016/j.ocemod.2012.12.003.

__ and J. Taylor, 2014: Modelling of partially-resolved oceanic symmetric instability. Ocean Modell., 82, 15-27, https:// doi.org/10.1016/j.ocemod.2014.07.006.

— B. Fox-Kemper, J. Taylor, and L. Thomas, 2017: Parameterization of frontal symmetric instabilities. I: Theory for resolved fronts. Ocean Modell., 109, 72-95, https://doi.org/ 10.1016/j.ocemod.2016.12.003.

Boccaletti, G., R. Ferrari, and B. Fox-Kemper, 2007: Mixed layer instability and restratification. J. Phys. Oceanogr., 37, 22282250, https://doi.org/10.1175/JPO3101.1.

Callies, J., and R. Ferrari, 2018a: Note on the rate of restratification in the baroclinic spindown of fronts. J. Phys. Oceanogr., 48, 1543-1553, https://doi.org/10.1175/JPO-D-17-0175.1.

$\longrightarrow$, and $-2018 \mathrm{~b}$ : Baroclinic instability in the presence of convection. J. Atmos. Sci., 48, 45-60, https://doi.org/10.1175/ JPO-D-17-0028.1.

Capet, X., J. C. McWilliams, M. J. Molemaker, and A. F. Shchepetkin, 2008: Mesoscale to submesoscale transition in the California Current System. Part I: Flow structure, eddy flux, and observational tests. J. Phys. Oceanogr., 38, 22562269, https://doi.org/10.1175/2007JPO3671.1.

Fox-Kemper, B., R. Ferrari, and R. Hallberg, 2008: Parameterization of mixed layer eddies. Part I: Theory and diagnosis. J. Phys. Oceanogr., 38, 1145-1165, https://doi.org/10.1175/ 2007JPO3792.1.

_ - and Coauthors, 2011: Parameterization of mixed layer eddies. III: Implementation and impact in global ocean climate simulations. Ocean Modell., 39, 61-78, https://doi.org/10.1016/ j.ocemod.2010.09.002.

Haine, T. W. N., and J. Marshall, 1998: Gravitational, symmetric, and baroclinic instability of the ocean mixed layer. J. Phys. Oceanogr., 28, 634-658, https://doi.org/10.1175/1520-0485(1998) $028<0634$ :GSABIO $>2.0 . \mathrm{CO} ; 2$.

Haney, S., B. Fox-Kemper, K. Julien, and A. Webb, 2015: Symmetric and geostrophic instabilities in the wave-forced ocean mixed layer. J. Phys. Oceanogr., 45, 3033-3056, https://doi.org/ 10.1175/JPO-D-15-0044.1.

Held, I. M., and D. G. Andrews, 1983: On the direction of the eddy momentum flux in baroclinic instability. J. Atmos. Sci., 40,
2220-2231, https://doi.org/10.1175/1520-0469(1983)040<2220: OTDOTE $>2.0 . \mathrm{CO} ; 2$.

Hoskins, B. J., 1974: The role of potential vorticity in symmetric stability and instability. Quart. J. Roy. Meteor. Soc., 100, 480 482, https://doi.org/10.1002/qj.49710042520.

James, I. N., 1987: Suppression of baroclinic instability in horizontally sheared flows. J. Atmos. Sci., 44, 3710-3720, https:// doi.org/10.1175/1520-0469(1987)044<3710:SOBIIH>2.0.CO;2.

_ , and L. J. Gray, 1986: Concerning the effect of surface drag on the circulation of a baroclinic planetary atmosphere. Quart. J. Roy. Meteor. Soc., 112, 1231-1250, https://doi.org/10.1002/ qj.49711247417.

Kuo, H. L., 1949: Dynamic instability of two-dimensional nondivergent flow in a barotropic atmosphere. J. Meteor., 6 , 105-122, https://doi.org/10.1175/1520-0469(1949)006<0105: DIOTDN $>2.0 . \mathrm{CO} ; 2$.

Lévy, M., R. Ferrari, P. J. S. Franks, A. P. Martin, and P. Rivière, 2012: Bringing physics to life at the submesoscale. Geophys. Res. Lett., 39, L14602, https://doi.org/10.1029/2012GL052756.

Mahadevan, A., A. Tandon, and R. Ferrari, 2010: Rapid changes in mixed layer stratification driven by submesoscale instabilities and winds. J. Geophys. Res., 115, C03017, https://doi.org/ 10.1029/2008JC005203.

Marshall, J., and R. A. Plumb, 2008: Atmosphere, Ocean and Climate Dynamics: An Introductory Text. Elsevier Academic Press, 344 pp.

McIntyre, M. E., 1970: On the non-separable baroclinic parallel flow instability problem. J. Fluid Mech., 40, 273-306, https:// doi.org/10.1017/S0022112070000174.

McWilliams, J. C., 2016: Submesoscale currents in the ocean. Proc. Roy. Soc. London, 472A, 20160117, https://doi.org/10.1098/ rspa.2016.0117.

Nakamura, N., 1993a: An illustrative model of instabilities in meridionally and vertically sheared flows. J. Atmos. Sci., 50, 357-376, https://doi.org/10.1175/1520-0469(1993)050<0357: AIMOII $>2.0 . \mathrm{CO} ; 2$.

_ 1993b: Momentum flux, flow symmetry, and the nonlinear barotropic governor. J. Atmos. Sci., 50, 2159-2179, https://doi.org/ 10.1175/1520-0469(1993)050<2159:MFFSAT>2.0.CO;2.

Ramachandran, S., A. Tandon, and A. Mahadevan, 2014: Enhancement in vertical fluxes at a front by mesoscale-submesoscale coupling. J. Geophys. Res. Oceans, 119, 8495-8511, https://doi.org/ 10.1002/2014JC010211.

Rhines, P. B., 1979: Geostrophic turbulence. Annu. Rev. Fluid Mech., 11, 401-441, https://doi.org/10.1146/annurev. fl.11.010179.002153.

Simmons, A. J., and B. J. Hoskins, 1978: The life cycles of some nonlinear baroclinic waves. J. Atmos. Sci., 35, 414-432, https://doi.org/10.1175/1520-0469(1978)035<0414:TLCOSN > 2.0.CO;2.

Stamper, M. A., and J. R. Taylor, 2017: The transition from symmetric to baroclinic instability in the Eady model. Ocean Dyn., 67, 65-80, https://doi.org/10.1007/s10236-016-1011-6.

Stone, P. H., 1966: On non-geostrophic baroclinic stability. J. Atmos. Sci., 23, 390-400, https://doi.org/10.1175/1520-0469(1966) 023<0390:ONGBS $>2.0$. CO; 2 .

__, 1972: On non-geostrophic baroclinic stability: Part III. The momentum and heat transports. J. Atmos. Sci., 29, 419-426, https://doi.org/10.1175/1520-0469(1972)029<0419: ONGBSP $>2.0 . \mathrm{CO} ; 2$.

Taylor, J. R., 2008: Numerical simulations of the stratified oceanic bottom boundary layer. Ph.D. thesis, University of California, San Diego, 230 pp. 
_- , and R. Ferrari, 2009: On the equilibrium of a symmetrically unstable front via a secondary shear instability. J. Fluid Mech., 622, 103-113, https://doi.org/10.1017/ S0022112008005272.

_- S. Bachman, M. Stamper, P. Hosegood, K. Adams, J.-B. Sallee, and R. Torres, 2018: Submesoscale Rossby waves on the Antarctic Circumpolar Current. Sci. Adv., 4, eaao2824, https://doi.org/10.1126/sciadv.aao2824.

Thomas, L. N., A. Tandon, and A. Mahadevan, 2008: Submesoscale processes and dynamics. Ocean Modeling in an Eddying Regime, Geophys. Monogr., Vol. 177, Amer. Geophys. Union, 17-38.
—, J. R. Taylor, E. A. D'Asaro, C. M. Lee, J. M. Klymak, and A. Shcherbina, 2016: Symmetric instability, inertial oscillations, and turbulence at the Gulf Stream front. J. Phys. Oceanogr., 46, 197-217, https://doi.org/10.1175/JPO-D-15-0008.1.

Vallis, G. K., and M. E. Maltrud, 1993: Generation of mean flows and jets on a beta plane and over topography. J. Phys. Oceanogr., 23, 1346-1362, https://doi.org/10.1175/1520-0485(1993) $023<1346$ :GOMFAJ $>2.0 . \mathrm{CO} ; 2$.

Whitt, D. B., and J. R. Taylor, 2017: Energetic submesoscales maintain strong mixed layer stratification during an autumn storm. J. Phys. Oceanogr., 47, 2419-2427, https://doi.org/ 10.1175/JPO-D-17-0130.1. 